\title{
Learning Hybrid Representation by Robust Dictionary Learning in Factorized Compressed Space
}

\author{
Jiahuan Ren, Zhao Zhang, Senior Member, IEEE, Sheng Li, Senior Member, IEEE, Yang Wang, Guangcan Liu, \\ Senior Member, IEEE, Shuicheng Yan, Fellow, IEEE, and Meng Wang
}

\begin{abstract}
In this paper, we investigate the robust dictionary learning (DL) to discover the hybrid salient low-rank and sparse representation in a factorized compressed space. A Joint Robust Factorization and Projective Dictionary Learning (J-RFDL) model is presented. The setting of J-RFDL aims at improving the data representations by enhancing the robustness to outliers and noise in data, encoding the reconstruction error more accurately and obtaining hybrid salient coefficients with accurate reconstruction ability. Specifically, J-RFDL performs the robust representation by DL in a factorized compressed space to eliminate the negative effects of noise and outliers on the results, which can also make the DL process efficient. To make the encoding process robust to noise in data, J-RFDL clearly uses sparse $L_{2,1}$-norm that can potentially minimize the factorization and reconstruction errors jointly by forcing rows of the reconstruction errors to be zeros. To deliver salient coefficients with good structures to reconstruct given data well, J-RFDL imposes the joint low-rank and sparse constraints on the embedded coefficients with a synthesis dictionary. Based on the hybrid salient coefficients, we also extend J-RFDL for the joint classification and propose a discriminative J-RFDL model, which can improve the discriminating abilities of learnt coefficients by minimizing the classification error jointly. Extensive experiments on public datasets demonstrate that our formulations can deliver superior performance over other state-of-the-art methods.
\end{abstract}

Index Terms-Hybrid salient representation, robust factorized compression, robust projective dictionary learning, classification

\section{INTRODUCTION}

$\mathrm{W}$ ITH the increasing complexity of contents, diversity of distribution and high-dimensionality of real data, how to represent data efficiently for subsequent classification or clustering still remains an important research topic [1-3][9][50]. To represent data, some feasible methods can be used, such as sparse representation (SR) by dictionary learning (DL) [4-8], low-rank coding [9-10][15][38-39] and matrix factorization [11] [12], which are inspired by the fact that high-dimensional data

- J. Ren is with the School of Computer Science and Technology, Soochow University, Suzhou 215006, China (e-mails: hmzry10086@outlook.com)

- Z. Zhang is with the Key Laboratory of Knowledge Engineering with Big Data (Hefei University of Technology), Ministry of Education, China; also with Soochow University, Suzhou, China. (e-mail: cszzhang@gmail.com)

- Y. Wang and M. Wang are now with the Key Laboratory of Knowledge Engineering with Big Data (Ministry of Education), Hefei University of Technology, Hefei, China. (e-mails: yeungwangresearch@gmail.com, eric.mengwang@gmail.com)

- $\mathrm{S}$. Li is with the Department of Computer Science, University of Georgia, Athens, GA 30602, USA (e-mail: sheng.li@uga.edu)

- G. Liu is with the School of Information and Control, Nanjing University of Information Science and Technology, Nanjing, China (e-mail: gcliu@nuist.edu.cn)

- S. Yan is with YITU Technology; also with the National University of Singapore, Singapore. (e-mail: shuicheng.yan@yitu-inc.com) can usually be characterized by applying a low-dimensional or compressed space in which the possible noise and redundant information can be removed in addition to preserving the useful information and important structures.

Dictionary learning (DL) algorithms mainly study the topics on seeking an over-complete dictionary for representation and classification, which can be roughly divided into unsupervised and discriminative ones. Unsupervised DL methods do not use any supervised prior information (e.g., label information of the training data) and mainly aim at minimizing the reconstruction error to produce a reliable dictionary and a set of informative coefficients. To represent data, SR approximates each sample by a linear combination of a few items from a dictionary [4], so the quality of learnt dictionary is a dominating factor for SR. To obtain a reliable dictionary, many unsupervised DL methods [20][47-48] have been proposed, of which $K$-Singular Value Decomposition (KSVD) [20] is one most representative model and it seeks a dictionary from the whole training set. Note that for unsupervised learning by factorization, Nonnegative Matrix Factorization (NMF) [11] and Concept Factorization (CF) [12] are two most popular ones, and they both aim at factorizing the original data into the product between a set of nonnegative basis vectors and the compressed new representation. CF model is a variant of NMF by expressing each sample with a linear combination of cluster centers [12]. Low-rank coding is also an important representation model, such as [9][13][34]. Several representative feature embedding based unsupervised low-rank representation (LRR) models are Inductive Robust Principal Component Analysis (IRPCA) [14], Latent LRR (LatLRR) [15] and Regularized LRR (rLRR) [16] that can explicitly obtain a low-rank projection to extract salient low-rank features from given data and remove noise at the same time. More recently, an Inductive Joint Low-Rank and Sparse Principal Feature Coding (I-LSPFC) model [18] was presented for enhancing the representations by jointly exploiting the low-rank and sparse feature spaces. Note that unsupervised models mainly consider representing data, but cannot process the classification task.

In contrast to the unsupervised DL methods, discriminative approaches make full use of label information of samples when available to improve the joint representation and classification abilities [21-22][25-31][38][40]. Existing supervised methods can be further divided into two categories. The first category aims at learning the overall dictionary and the representative methods are Discriminative KSVD (D-KSVD) [25] and Label Consistent KSVD (LC-KSVD) [27]. D-KSVD obtains a desired dictionary of supporting the inter-class discrimination, while LC-KSVD learns a discriminative dictionary by adding a label consistent constraint and combining it with reconstruction error and classification error to form a unified model. The second 


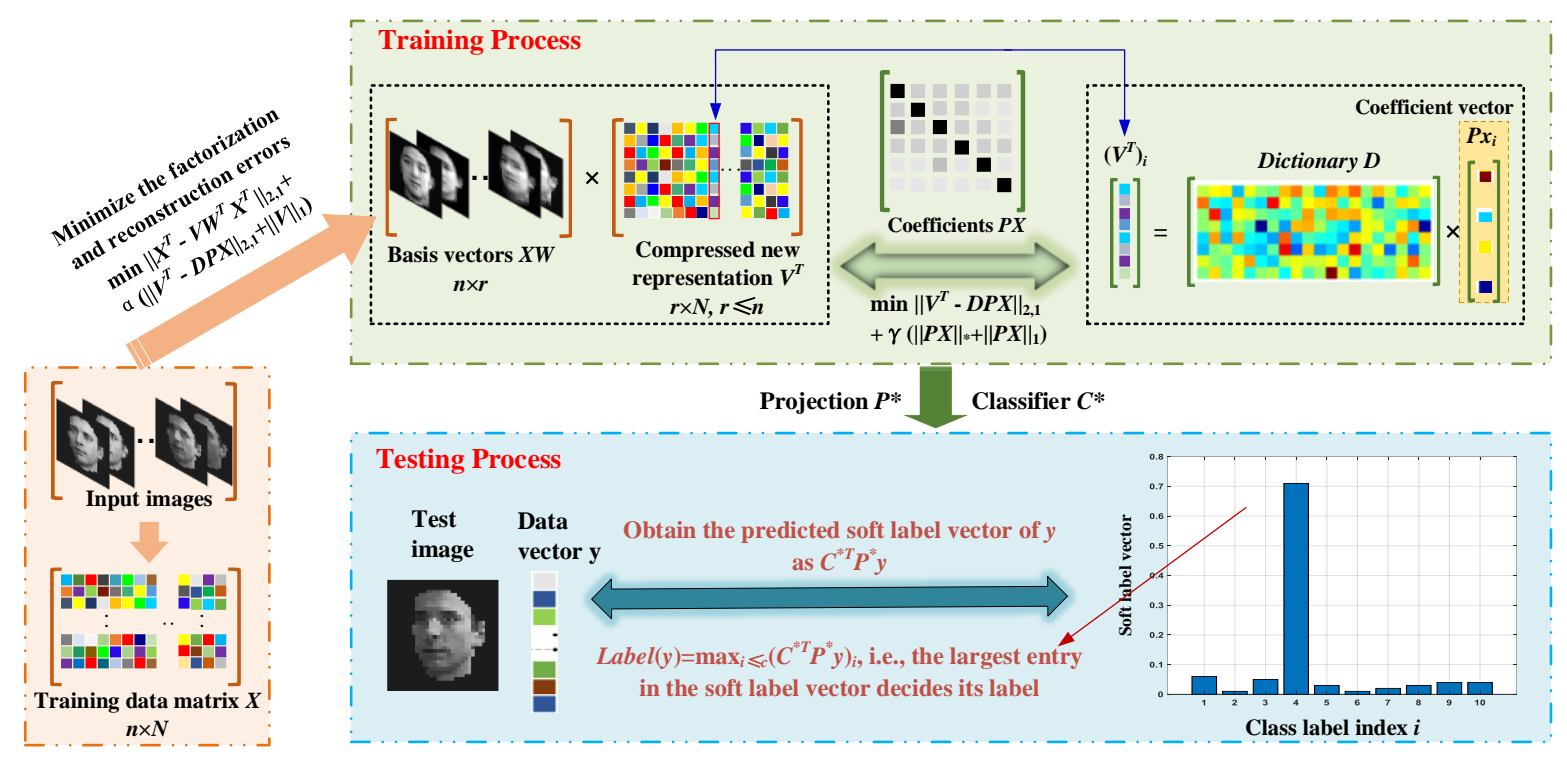

Fig. 1: The schematic diagram of our proposed J-RFDL and DJ-RFDL frameworks for training and testing.

category aims at learning the category-specific dictionaries to improve the discrimination by assigning each sub-dictionary to a single subject class, i.e., structured dictionary learning. In this category, popular methods are Low-rank Shared DL (LRSDL) [52], Analysis Discriminative Dictionary Learning (ADDL) [22], Dictionary Learning with Structured Incoherence (DLSI) [21] and Projective Dictionary Pair Learning (DPL) [17]. DPL aims to obtain a structured synthesis dictionary and an analysis dictionary jointly, and ADDL is based on the idea of DPL and it aims to compute a structured analysis discriminative dictionary and an analysis multiclass classifier.

It is worth noting that aforementioned existing methods still suffer from some shortcomings. First, most existing methods obtain the sparse representations and dictionaries in the original input space that usually consists of various noise, redundant information and even errors, so the data representation ability may be potentially degraded. Second, most existing methods encode the reconstruction error using the Frobenius-norm that is sensitive to noise and outliers, so the resulted reconstruction error may be inaccurate in reality. Thus, it will be helpful to present a robust DL approach that can work in a compressed feature subspace from which redundant information, noise and outliers are removed. Third, existing models usually compute the coefficients of each new sample by using a time-consuming reconstruction process with a well-trained dictionary, such as D-KSVD and LC-KSVD. Although DPL and ADDL aim to address this issue by calculating a synthesis dictionary jointly, they both did not consider regularizing the synthesis dictionary to obtain the salient low-rank and sparse coefficients. Most real data can usually be represented using a sparse and/or low-rank subspace due to the intrinsic low-dimensional characteristics [4-10]. Thus, without considering the joint sparse and low-rank constraints properly, the resulted structures of the coefficients may not represent the given data appropriately and accurately.

Motivated by the above existing shortcomings, in this paper we propose a new robust dictionary learning framework called Joint Robust Factorization and Projective Dictionary Learning (J-RFDL) for recovering the joint salient low-rank and sparse coefficients in a compressed factorized feature subspace. To be specific, J-RFDL integrates the robust factorization, projective DL and the embedded low-rank \& sparse coding into a unified framework. We describe the major contributions as follows:

(1) J-RFDL performs the robust representation learning in a factorized compressed space in which unfavorable features and redundant information are removed from original data.

(2) To make the coding process robust to noise and outliers in data, and potentially reduce the factorization and reconstruction errors, J-RFDL uses the sparse and robust $L_{2,1} 1$-norm [35-37].

(3) J-RFDL can obtain the salient low-rank and sparse codes for accurate representations. Based on the salient coefficients, a Discriminative J-RFDL (DJ-RFDL) is also presented.

The paper is outlined as follows. In Section II, we review the related work briefly. In Sections III and IV, we present J-RFDL and DJ-RFDL, respectively. Section V shows the connections with other methods. Section VI shows the experimental results and analysis. Finally, the paper is concluded in Section VII.

\section{RELATED WORK}

\section{A. Nonnegative Concept Factorization (CF)}

Given a set of samples $X=\left[x_{1}, x_{2} \cdots, x_{N}\right] \in \mathbb{R}^{n \times N}$, where $x_{i} \in \mathbb{R}^{n}$ is a sample that is represented by an $n$-dimensional vector, $n$ is the original dimensionality and $N$ is the number of samples. Note that the nonnegative factorization can be mathematically represented as finding two nonnegative matrix factors $U \in \mathbb{R}^{n \times r}$ and $V^{T} \in \mathbb{R}^{r \times N}$ whose product can best approximate $X$, i.e.,

$$
X \approx U V^{T} \text {, where } U \geq 0, V \geq 0,
$$

where each column of $U$ is the basis vector that captures the higher-level features of samples, and $V^{T}$ is low-dimensional representation of original data. From this viewpoint, the matrix factorization can be regarded as the dimensionality reduction method since it reduces the dimension from $n$ to $r$. NMF and CF are the most popular factorization methods and the nonnegative constraints can learn the parts-based representation [11-12][42]. But how to perform NMF in reproducing kernel Hilbert space so that the powerful kernel method can be applied [12][42] is unclear. CF is proposed to solve this issue, and the advantage of 
$\mathrm{CF}$ is that it can be applied to any representation space, which is implemented by representing each base $u_{r}$ by using a linear combination of samples, i.e., $u_{r}=\sum w_{j r} x_{j}$.

That is, CF aims to decompose $X$ and satisfy the equation $X \approx X W V^{T}$ by minimizing the following cost function:

$$
\phi=\left\|X-X W V^{T}\right\|_{F}^{2}, \text { s.t. } W \geq 0, V \geq 0,
$$

where $r$ is the rank of the factorization or the dimension of the reduced feature space, $V^{T}=\left[v_{i j}\right] \in \mathbb{R}^{r \times N}$ and $W=\left[w_{i j}\right] \in \mathbb{R}^{N \times r}$ with $w_{i j} \geq 0$ and $v_{i j} \geq 0$. CF applies an iteration strategy to update $V$ and $W$. Specifically, in the $(k+1)$-th iteration, $V$ and $W$ can be updated using the following multiplicative updating rules:

$$
w_{j r}^{k+1}=w_{j r}^{k} \frac{(A V)_{j r}}{\left(A W V^{T} V\right)_{j r}}, v_{j r}^{k+1}=v_{j r}^{k} \frac{(A W)_{j r}}{\left(V W^{T} A W\right)_{j r}},
$$

where $A=X^{T} X$ is an auxiliary matrix.

\section{B. Dictionary Learning and Dictionary Pair Learning (DPL)}

Given the data matrix $X$, the standard DL algorithms solve the following general framework to compute the reconstructive dictionary $D$ and sparse representation $S$ of $X$ :

$$
\langle D, S\rangle=\arg \min _{D, S}\|X-D S\|_{F}^{2}+\kappa\|S\|_{p} \text {, s.t. }\left\|d_{i}\right\|_{2}^{2} \leq 1,
$$

where $\kappa>0$ denotes a scalar constant, $D=\left[d_{1}, \cdots d_{K}\right] \in \mathbb{R}^{n \times K}$ is an overall dictionary obtained by minimizing the reconstruction error $\|X-D S\|_{F}^{2}$. $\|S\|_{p}$ is the $L_{\mathrm{p}}$-norm regularized term, where $S=\left[s_{1} \cdots s_{N}\right] \in \mathbb{R}^{K \times N}$ is the coding coefficients of $X$ and usually the parameter $p$ is usually set to 0 or 1 to make the learned coefficients sparse. However, the computations of $L_{0}$-norm and $L_{1}$-norm are usually time-consuming in reality.

Suppose that label information of training set is known, i.e., the sample set can be expressed as $X=\left[X_{1}, \cdots X_{l} \cdots, X_{N}\right] \in \mathbb{R}^{n \times N}$, where $X_{l} \in \mathbb{R}^{n \times N_{l}}$ is the training set of class $l$ and $N_{l}$ is the number of samples in the class $l$. To avoid the costly computation of $S$, DPL learns a synthesis dictionary $D$ and an analysis dictionary $P$ to approximate the coding coefficients without using costly $L_{0}$-norm $/ L_{1}$-norm constraint by solving the following problem:

$$
\langle P, D\rangle=\arg \min _{P, D} \sum_{i=1}^{c}\left\|X_{i}-D_{i} P_{i} X_{i}\right\|_{F}^{2}+\lambda\left\|P_{i} \bar{X}_{i}\right\|_{F}^{2}, \text { s.t. }\left\|d_{j}\right\|_{2}^{2} \leq 1,
$$

where $\bar{X}_{i}$ is the complementary data matrix of $X_{i}$ in $X . D_{i}$ is a structured synthesis sub-dictionary of class $i, P_{i}$ is a structured analysis sub-dictionary of class $i, D_{i} \in \mathbb{R}^{n \times K}, P_{i} \in \mathbb{R}^{K \times n}$ and $K$ is the dictionary size. Clearly, by setting $P_{i} X_{q} \approx 0, \forall q \neq i$, the coefficient matrix $P X$ will be nearly block diagonal. $d_{j}$ is the $j$-th atom of dictionary $D$, and the constraint $\left\|d_{j}\right\|_{2}^{2} \leq 1$ on $d_{j}$ is to avoid the trivial solution of $P_{i}=0$ and make the algorithm stable.

\section{JoINT RoBUSt FACTORIZATION AND PROJECTIVE DICTIONARY LEARNING FRAMEWORK (J-RFDL)}

\section{A. The Objective Function}

We present the problem formulation of our J-RFDL that aims at learning the hybrid salient low-rank and sparse representations by robust dictionary learning in factorized compressed space. Specifically, it improves the data representations by enhancing the robustness to outliers and noise in data during both DL and matrix factorization processes, encoding the factorization and reconstruction errors accurately with row sparse constraint and delivering the hybrid salient representation coefficients with superior reconstruction power. To define the problem, J-RFDL integrates the robust factorization, robust dictionary learning and embedded low-rank \& sparse representation learning into a unified model. By combining the factorization error $f\left(W, V^{T}\right)$ with the sample reconstruction error $g(D, P)$, we can define the following unified problem for our J-RFDL:

$$
\min _{W, V, D, P} f\left(W, V^{T}\right)+g(D, P) \text {, s.t. } W, V \geq 0, e^{T} D=e^{T},
$$

where $W$ is also a nonnegative matrix as in CF, $V^{T}$ is learnt new representation, $D$ is a dictionary and $P$ is a projection matrix to obtain the embedded low-rank \& sparse coefficients $P X$. Clearly, $g(D, P)$ is the reconstruction error by robust dictionary learning over $V^{T}$. So, performing DL in the reduced feature space can be more efficient, especially when the dimension of $X$ is high. The sum-to-one constraint $e^{T} D=e^{T}$ can also prevent the big values of $D$ and make the problem stable, where $e$ is a vector of ones. $f\left(W, V^{T}\right)$ and $g(D, P)$ will be introduced shortly. The above problem can be alternated between the following steps:

\section{(1) Robust dictionary learning in the factorized compressed space for hybrid low-rank and sparse representation}

We first fix the new representation $V^{T}$ by robust sparse concept factorization to update the dictionary $D$ and hybrid low-rank $\&$ sparse coding coefficients $P X$ from the following problem:

$$
\min _{D, P} g(D, P)=\alpha\left\|V^{\mathrm{T}}-D P X\right\|_{2,1}+\gamma\left(\|P X\|_{*}+\|P X\|_{1}\right) \text {, s.t. } e^{T} D=e^{T},
$$

where $\left\|V^{\mathrm{T}}-D P X\right\|_{2,1}$ is the $L_{2,1}$-norm based reconstruction error term for robust DL. $L_{2,1}$-norm can minimize the reconstruction error as much as possible because it tends to make rows of the reconstruction error $V^{\mathrm{T}}-D P X$ to be zeros [35]. In other words, minimizing the reconstruction error can enable $D P X$ to best approximate $V^{\mathrm{T}}$. Besides, the $L_{2,1}$-norm based reconstruction is so robust to noise and outliers [35]. To make the coefficients $P X$ satisfy the hybrid low-rank and sparse properties to enhance the representation ability, the Nuclear-norm and $L_{l}$-norm are jointly regularized on $P X$, i.e., $\|P X\|_{*}+\|P X\|_{1}$, which is motivated by the LSPFC algorithm [18]. But note that J-RFDL differs from LSPFC in two aspects. First, LSPFC is a low-rank coding model for decomposing given data into a salient feature part and a sparse error part, while J-RFDL is a robust dictionary learning method. Second, LSPFC encodes salient low-rank and sparse features, while J-RFDL obtains the hybrid low-rank and sparse coefficients for the data representations.

\section{(2) Robust sparse concept factorization for compression:}

After the $D$ and projection $P$ are obtained, we can learn the new sparse representation $V^{T}$ by solving the following $L_{2,1}$-norm and $L_{1}$-norm regularized robust concept factorization problem:

$$
\min _{W, V} f\left(W, V^{T}\right)=\left\|X^{\mathrm{T}}-V W^{T} X^{T}\right\|_{2,1}+\alpha\left(\left\|V^{\mathrm{T}}-D P X\right\|_{2,1}+\|V\|_{1}\right),
$$

s.t. $W, V \geq 0$, where $\left\|X^{\mathrm{T}}-V W^{T} X^{T}\right\|_{2,1}$ is the $L_{2,1}$-norm based factorization error, $\|V\|_{1}$ is the $L_{1}$-norm constraint so that learnt $V^{T}$ holds the sparse properties, and the product of $X, W$ and $V^{T}$ is an approximation to $X$. The $L_{2,1}$-norm can also minimize the error as much as possible by forcing many rows of the error to 
be zeros [35]. It is clear that $V^{T}$ is obtained via minimizing the joint reconstruction error $\left\|X^{\mathrm{T}}-V W^{T} X^{T}\right\|_{2,1}+\alpha\left\|V^{\mathrm{T}}-D P X\right\|_{2,1}$, i.e., the robust projective DL process also contributes to improving the representation ability of $V^{T}$. In other words, the optimization of $V^{T}$ is also associated with the pre-calculated dictionary $D$ and hybrid coefficients $P X$ in the last step.

By combining the equations in Eqs.(5), (6) and (7), the final objective function of our J-RFDL is formulated as

$$
\begin{aligned}
& \min _{D, P, W, V}\left\|X^{T}-V W^{T} X^{T}\right\|_{2,1}+\alpha\left(\left\|V^{\mathrm{T}}-D P X\right\|_{2,1}+\|V\|_{1}\right)+\gamma\left(\|P X\|_{*}+\|P X\|_{1}\right), \\
& \text { s.t. } W, V \geq 0, e^{T} D=e^{T}
\end{aligned}
$$

where the optimal $P^{*} X$ is also regarded the "hybrid lowest-rank and sparsest representation" of the original data. The schematic diagram of our J-RFDL framework is shown in Fig.1, where we present the training and testing phases. Next, we describe the optimization procedures of our J-RFDL.

\section{B. Optimization}

The variables in the objective function of J-RFDL depend on each other, so we follow the common procedures to solve it by an alternate strategy and use the inexact Augmented Lagrange Multiplier (ALM) method [19] for efficiency. We first convert the objective function into the following equivalent one:

$$
\begin{aligned}
& \min _{\substack{D, P, W, V \\
J, S, F}}\left\|X^{\mathrm{T}}-V W^{T} X^{T}\right\|_{2,1}+\alpha\left(\left\|V^{\mathrm{T}}-D P X\right\|_{2,1}+\|F\|_{1}\right)+\gamma\left(\|J\|_{*}+\|S\|_{1}\right) \\
& \text { s.t. } V=F, P X=J, P X=S, W \geq 0, V \geq 0, e^{T} D=e^{T}
\end{aligned}
$$

from which the Lagrange function $\wp$ can be constructed as

$$
\begin{aligned}
\wp= & \left\|X^{T}-V W^{T} X^{T}\right\|_{2,1}+\alpha\left(\left\|V^{T}-D P X\right\|_{2,1}+\|F\|_{1}\right)+\gamma\left(\|J\|_{*}+\|S\|_{1}\right) \\
& +\left\langle Y_{1}, V-F\right\rangle+\left\langle Y_{2}, P X-J\right\rangle+\left\langle Y_{3}, P X-S\right\rangle \\
& +\frac{\mu}{2}\left(\|V-F\|_{F}^{2}+\|P X-J\|_{F}^{2}+\|P X-S\|_{F}^{2}\right)
\end{aligned}
$$

with respect to $W, V \geq 0, e^{T} D=e^{T}$, where $Y_{1}, Y_{2}, Y_{3}$ are Lagrange multipliers, and $\mu$ is a positive weighting parameter. By using the inexact ALM, our J-RFDL updates the variables by

$$
\begin{aligned}
& \left(D_{k+1}, P_{k+1}, W_{k+1}, V_{k+1}, J_{k+1}, S_{k+1}, F_{k+1}\right)=\arg \min _{D, P, W, V, J, S, F} \wp_{k} \\
& Y_{1}^{k+1}=Y_{1}^{k}+\mu\left(V_{k}-F_{k}\right), Y_{2}^{k+1}=Y_{2}^{k}+\mu\left(P_{k} X-J_{k}\right) \\
& Y_{3}^{k+1}=Y_{3}^{k}+\mu\left(P_{k} X-S_{k}\right), \mu_{k+1}=\min \left(\eta \mu_{k}, \max _{\mu}\right)
\end{aligned}
$$

Then, the optimization of J-RFDL can be detailed as follows.

1) Fix others, update $J, S, F$ :

We first update the auxiliary variable $J$. By removing the terms irrelevant to $J$, we can update $J_{k+1}$ at the $(k+1)$-th iteration as

$$
J_{k+1}=\arg \min _{J} \frac{\gamma}{\mu_{k}}\|J\|_{*}+\frac{1}{2}\left\|J-\left(P_{k} X+Y_{2}^{k} / \mu_{k}\right)\right\|_{F}^{2} .
$$

Following the procedure for solving the Nuclear-norm based problem [19], $J$ can be updated by SVD. More specifically, let $\mho_{\varepsilon}[x]=\operatorname{sgn}(x) \max (|x|-\varepsilon, 0)$ be the shrinkage operator, we can compute $J_{k+1}$ by the singular value thresholding algorithm as $J_{K=1}=M_{J} \mho_{\gamma / \mu}\left[\sum_{J}\right] Q_{J}^{T}$, where $M_{J} \sum_{J} Q_{J}^{T}$ represents the SVD of $P_{k} X+Y_{2}^{k} / \mu_{k}$. Note that the updating rules of both $S$ and $F$ are similarly as solving $J$ by the scalar shrinkage operator [19] as

$$
\begin{gathered}
S_{k+1}=\arg \min _{S} \frac{\gamma}{\mu_{k}}\|S\|_{1}+\frac{1}{2}\left\|S-\left(P_{k} X+Y_{3}^{k} / \mu_{k}\right)\right\|_{F}^{2}, \\
F_{k+1}=\arg \min _{F} \frac{\alpha}{\mu_{k}}\|F\|_{1}+\frac{1}{2}\left\|F-\left(V_{k}+Y_{1}^{k} / \mu_{k}\right)\right\|_{F}^{2} .
\end{gathered}
$$

\section{2) Fix others, update $D, P$ :}

With the other variables fixed, we can easily both $D$ and $P$ by performing robust dictionary learning. By removing the terms that are irrelevant to $D$ and $P$ from $\wp$, we have

$$
\begin{gathered}
\min _{D, P} \alpha\left\|V^{\mathrm{T}}-D P X\right\|_{2,1}+\left\langle Y_{2}, P X-J\right\rangle+\left\langle Y_{3}, P X-S\right\rangle \\
+\frac{\mu}{2}\left(\|P X-J\|_{F}^{2}+\|P X-S\|_{F}^{2}\right), \text { s.t. } e^{T} D=e^{T}
\end{gathered}
$$

Based on the definition of $L_{2,1}$-norm [35], we can easily have $\left\|V^{\mathrm{T}}-D P X\right\|_{2,1}=2 \operatorname{tr}\left(\left(V^{\mathrm{T}}-D P X\right)^{\mathrm{T}} Q\left(V^{\mathrm{T}}-D P X\right)\right)$, where $Q$ denotes a diagonal matrix with entries $q_{i i}=1 /\left(2\left\|\chi^{i}\right\|_{2}\right)$ and $\chi^{i}$ is $i$-th row vector of matrix $V^{\mathrm{T}}-D P X$. Suppose that each vector $\chi^{i} \neq 0$, the above problem can be approximated as

$$
\begin{gathered}
\min _{D, P, Q} \operatorname{tr}\left(\alpha\left(V^{\mathrm{T}}-D P X\right)^{\mathrm{T}} Q\left(V^{\mathrm{T}}-D P X\right)\right)+\left\langle Y_{2}, P X-J\right\rangle \\
+\left\langle Y_{3}, P X-S\right\rangle+\frac{\mu}{2}\left(\|P X-J\|_{F}^{2}+\|P X-S\|_{F}^{2}\right)
\end{gathered} .
$$

By taking the derivative of the above equation w.r.t. $D$ and zeroing the derivative, we can update the dictionary $D_{k+1}$ as

$$
D_{k+1}=Q_{k}^{-1}\left(Q_{k} V_{k}^{T} X^{T} P_{k}^{T}\right)\left(P_{k} X X^{T} P_{k}^{T}\right)^{-1}, e^{T} D_{k+1}=e^{T} .
$$

Similarly, by taking the derivative w.r.t. $P$ and setting it to zero, we can update the projection $P_{k+1}$ as

$$
P_{k+1}=\left(2 \alpha D_{k+1}{ }^{T} Q_{k} D_{k+1}+2 \mu_{k} I\right)^{-1} L_{k}\left(X X^{T}+\tau I\right)^{-1} .
$$

where $L_{k}=\left(2 \alpha\left(X V_{k} Q_{k} D_{k+1}\right)^{T}-Y_{2}^{k} X^{T}-Y_{3}^{k} X^{T}+\mu_{k} J_{k} X^{T}+\mu_{k} S_{k} X^{T}\right)$, and $\tau I$ with a small $\tau$ is to avoid the singularity issue.

After both $D$ and $P$ are obtained, we can update the entries of the diagonal matrix $Q$ as $\left(q_{i i}\right)_{k+1}=1 /\left(2\left\|\chi_{k+1}^{i}\right\|_{2}\right)$, where $\chi_{k+1}^{i}$ is $i$-th row of matrix $V_{k+1}^{T}-D_{k+1} P_{k+1} X$.

\section{3) Fix others, update $W$ and $V$ :}

We update $W$ and $V$ by performing the robust sparse concept factorization. After removing the irrelevant terms, we have

$$
\begin{gathered}
\min _{W, V} \wp(W, V)=\left\|X^{T}-V W^{T} X^{T}\right\|_{2,1}+\alpha\left\|V^{T}-D P X\right\|_{2,1} \\
+\left\langle Y_{1}, V-F\right\rangle+\frac{\mu}{2}\|V-F\|_{F}^{2}, \quad \text { s.t. } W, V \geq 0
\end{gathered} .
$$

For the optimization of $W$ and $V$, we employ the iterative multiplicative updating rules to obtain the local optima. By the matrix expression and the definition of $L_{2,1}$-norm [35], we first transform Eq.(19) into the following equivalent one:

$$
\begin{aligned}
\min _{W, V, Q, G} \wp(W, V, Q, G)= & \operatorname{tr}\left(\left(X^{T}-V W^{T} X^{T}\right)^{\mathrm{T}} G\left(X^{T}-V W^{T} X^{T}\right)\right) \\
& +\operatorname{tr}\left(\alpha\left(V^{\mathrm{T}}-D P X\right)^{\mathrm{T}} Q\left(V^{\mathrm{T}}-D P X\right)\right) \\
& +\operatorname{tr}\left(Y_{1}^{T}(V-F)\right)+\frac{\mu}{2} \operatorname{tr}\left((V-F)^{T}(V-F)\right)
\end{aligned}
$$

where $G$ is a diagonal matrix with entries $g_{i i}=1 /\left(2\left\|\psi^{i}\right\|_{2}\right)$ and $\psi^{i}$ is $i$-th row vector of the matrix $X^{T}-V W^{T} X^{T}$. Note that the above problem holds if each $\psi^{j} \neq 0$ and $\psi^{j} \neq 0$. Let $\tau_{i, j}$ and $\zeta_{i, j}$ 


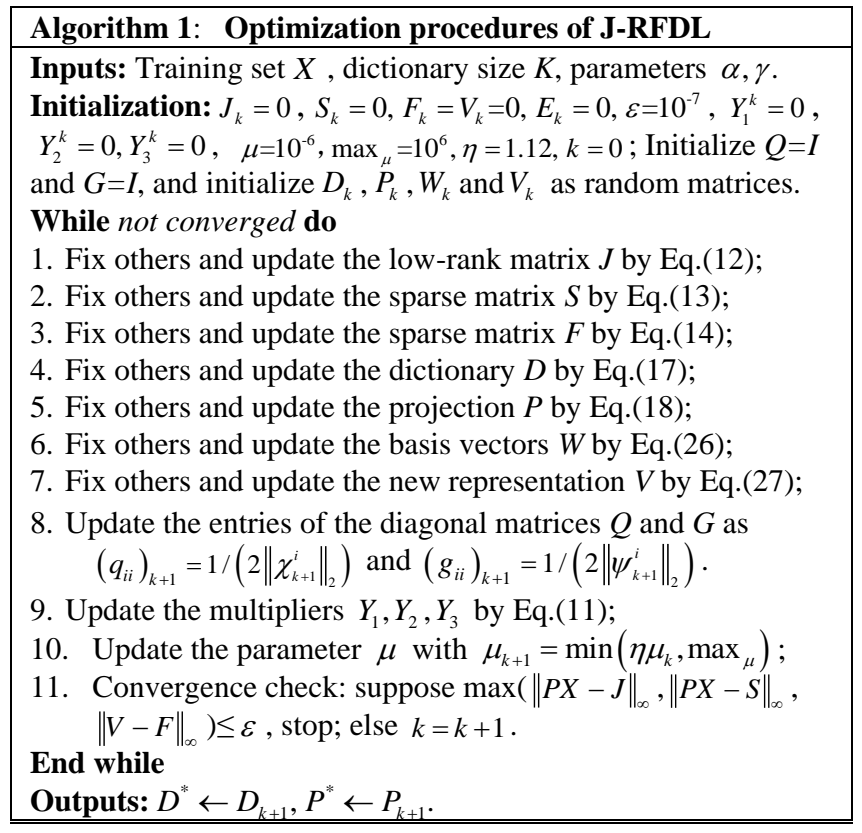

be the Lagrange multipliers for constraints $w_{i, j} \geq 0$ and $v_{i, j} \geq 0$. By denoting $\tau=\left[\tau_{i, j}\right]$ and $\varsigma=\left[\varsigma_{i, j}\right]$, the Lagrange function $\mathfrak{I}$ of the above problem can be obtained as

$$
\begin{aligned}
\mathfrak{I}= & \operatorname{tr}\left(\left(X^{T}-V W^{T} X^{T}\right)^{\mathrm{T}} G\left(X^{T}-V W^{T} X^{T}\right)\right) \\
& +\operatorname{tr}\left(\alpha\left(V^{\mathrm{T}}-D P X\right)^{\mathrm{T}} Q\left(V^{\mathrm{T}}-D P X\right)\right) \\
& +\operatorname{tr}\left(Y_{1}^{T}(V-F)\right)+\frac{\mu}{2} \operatorname{tr}\left((V-F)^{T}(V-F)\right)+\operatorname{tr}\left(\tau W^{T}\right)+\operatorname{tr}\left(\varsigma V^{T}\right)
\end{aligned}
$$

By taking the partial derivatives w.r.t. $W$ and $V$, we have

$$
\begin{aligned}
\partial \mathfrak{I} / \partial W & =-2 X^{T} X G V+2 X^{T} X W V^{T} G V+\tau, \\
\partial \Im / \partial V= & -2 G X^{T} X W+2 G V W^{T} X^{T} X W \\
& +\alpha\left(2 V Q-2 X^{T} P^{T} D^{T} Q\right)+Y_{1}+\mu(V-F)+\varsigma
\end{aligned} .
$$

By using the Kuhn-Tucker conditions $\tau_{i, j} w_{i, j}=0, \varsigma_{i, j} v_{i, j}=0$, we can obtain the following equations:

$$
\begin{gathered}
\left(X^{T} X W V^{T} Q V\right)_{i, j} w_{i, j}-\left(X^{T} X Q V\right)_{i, j} w_{i, j}=0, \\
\left(2 G V W^{T} X^{T} X W+2 \alpha V Q+Y_{1}+\mu V\right)_{i, j} v_{i, j}- \\
\left(2 G X^{T} X W+2 \alpha X^{T} P^{T} D^{T} Q+\mu F\right)_{i, j} v_{i, j}=0
\end{gathered} .
$$

These equations lead to the following updating rules:

$$
\begin{gathered}
w_{i, j} \leftarrow w_{i, j} \frac{\left(X^{T} X G V\right)_{i, j}}{\left(X^{T} X W V^{T} G V\right)_{i, j}}, \\
v_{i, j} \leftarrow v_{i, j} \frac{\left(2 G X^{T} X W+2 \alpha X^{T} P^{T} D^{T} Q+\mu F\right)_{i, j}}{\left(2 G V W^{T} X^{T} X W+2 \alpha V Q+Y_{1}+\mu V\right)_{i, j}} .
\end{gathered}
$$

After both $W$ and $V$ are obtained, we can update the entries of the diagonal matrix $G$ as $\left(g_{i i}\right)_{k+1}=1 /\left(2\left\|\psi_{k+1}^{i}\right\|_{2}\right)$, where $\psi_{k+1}^{i}$ is $i$-th row of $X_{k+1}^{T}-V_{k+1} W_{k+1}{ }^{T} X^{T}$. For complete presentation of the optimization procedures of our J-RFDL, we summarize them in Algorithm 1, where diagonal matrices $Q$ and $G$ are initialized to be the identity matrices as suggested in [24][35] that has shown that this kind of regularization can generally perform well. Note that an early version of this work has been presented in [59]. This paper also presents a discriminant extension, provides the detailed analysis of the formulation, and conducts a thorough evaluation on the tasks of representation and classification.

\section{Computational Time Complexity}

In the problem of our J-RFDL, the variables $D, P, W$ and $V$ are updated alternately. In each iteration, the time complexities of updating $D, P, W, V$ can be calculated. We employ the big $O$ notation to describe the time complexity as [46]. For the robust factorization error $f\left(W, V^{T}\right)$ and the rules in Eqs.(26-27), our J-RFDL has the same computational time complexity as CF for updating $W$ and $V$, i.e., $O\left(N^{2} r\right)$, where $N$ is the number of samples in $X$ and $r$ is the dimension of the new representation $V$. For the reconstruction error $g(D, P)$, the time complexities of updating $P$ and $D$ are $O\left(K^{3}+n N r+n^{3}\right)$ and $O\left(r^{3}+r N n+K^{3}\right)$ in each iteration. Thus, $O\left(k\left(K^{3}+n N r+n^{3}\right)\right)$ is the overall cost of our J-RFDL, when the updates stop after $k$ iterations.

\section{DISCRIMINATIVE J-RFDL (DJ-RFDL) FRAMEWORK}

Because J-RFDL focuses on the unsupervised representation [15-16][32-33] and has to seek an extra classifier by using the coefficients, but this cannot minimize the representation and classification errors jointly. To address this issue, we extend J-RFDL for joint classification by combining the classification error based on the hybrid salient coefficients and derive a more general model termed Discriminant J-RFDL (DJ-RFDL).

\section{A. Problem Formulation of DJ-RFDL}

When label information $\Gamma=\left[l\left(x_{1}\right), l\left(x_{2}\right), \cdots l\left(x_{N}\right)\right]$ of training set $X$ is available, where $l\left(x_{i}\right)$ is the label of each training data $x_{i}$, we can include a $L_{2,1}$-norm based regressive classification error $\left\|H^{\mathrm{T}}-X^{\mathrm{T}} P^{\mathrm{T}} C\right\|_{2,1}$ based on the salient coefficients $P X$ into the problem of J-RFDL for the simultaneous minimization, where $C \in \mathbb{R}^{K \times c}$ is a predictive classifier, $c$ is the number of classes and $C^{T} P x_{i}$ is the estimated soft label vector of each $x_{i}$ by $C$. That is, DJ-RFDL integrates the robust hybrid salient representation via dictionary learning and robust classifier learning. Note that this operation can ensure the hybrid $P X$ to be joint optimal to predict the labels of samples. The objective function of DJ-RFDL is

$$
\begin{aligned}
\min _{D, P, W, V, C}\left\|X^{T}-V W^{T} X^{T}\right\|_{2,1}+\alpha\left(\left\|V^{\mathrm{T}}-D P X\right\|_{2,1}+\|V\|_{1}\right) \\
+\gamma\left(\|P X\|_{*}+\|P X\|_{1}\right)+\beta\left(\left\|H^{\mathrm{T}}-X^{\mathrm{T}} P^{\mathrm{T}} C\right\|_{2,1}+\|C\|_{F}^{2}\right),
\end{aligned}
$$

s.t. $W, V \geq 0$

where $H=\left[h_{1}, h_{2}, \ldots h_{N}\right] \in \mathbb{R}^{c \times N}$ is a pre-defined label matrix of all training samples based on the labels $\Gamma=\left[l\left(x_{1}\right), l\left(x_{2}\right), \cdots l\left(x_{N}\right)\right]$, $h_{j}$ is the initial label vector of sample $x_{j}, h_{i, j}$ is the $i$-th entry of the column vector $h_{j}$ and $h_{i, j}=1$ if $x_{j}$ belongs to the class $i$ [18], $1 \leq i \leq c$. The $L_{2,1}$-norm on classification error can enable the metric to be robust to outliers and noise [24][35][37], and the use of $L_{2,1}$-norm on the classification error essentially assumes that some classes have larger classification errors than others. The classification error can be reduced, since $L_{2,1}$-norm can also make many rows of the classification error shrink to zeros [24] [35]. Clearly, DJ-RFDL is more general than J-RFDL. Next, we describe its optimization procedures. 


\section{B. Optimization}

Due to the convexity of the problem of DJ-RFDL, we also use the inexact ALM method [19] to optimize its objective function. Let $E=H^{T}-X^{T} P^{T} C$ be the classification error, we can convert Eq.(28) into the following equivalent one:

$$
\begin{aligned}
& \min _{\substack{D, P, W, V, J, S, F, C, E}}\left\|X^{\mathrm{T}}-V W^{T} X^{T}\right\|_{2,1}+\alpha\left(\left\|V^{\mathrm{T}}-D P X\right\|_{2,1}+\|F\|_{1}\right) \\
& \qquad+\gamma\left(\|J\|_{*}+\|S\|_{1}\right)+\beta\left(\|E\|_{2,1}+\|C\|_{F}^{2}\right) \\
& \text { s.t. } V=F, P X=J, P X=S, H^{T}=X^{T} P^{T} C+E, W \geq 0, V \geq 0
\end{aligned}
$$

The augmented Lagrange function can be similarly defined as

$$
\begin{aligned}
\Omega & =\wp+\beta\left(\|E\|_{2,1}+\|C\|_{F}^{2}\right)+\left\langle Y_{4}, H^{T}-X^{T} P^{T} C-E\right\rangle \\
& +\frac{\mu}{2}\left(\left\|H^{T}-X^{T} P^{T} C-E\right\|_{F}^{2}\right), \text { s.t. } W, V \geq 0
\end{aligned}
$$

where $\wp$ is the Lagrange function of J-RFDL. By the inexact ALM, DJ-RFDL updates the variables alternately as:

$$
\begin{aligned}
& \left(D_{k+1}, P_{k+1}, W_{k+1}, V_{k+1}, C_{k+1}, J_{k+1}, S_{k+1}, F_{k+1}, E_{k+1}\right)=\arg \min _{D, P, W, V, C, J, S, F} \Omega \\
& Y_{1}^{k+1}=Y_{1}^{k}+\mu\left(V_{k}-F_{k}\right), Y_{2}^{k+1}=Y_{2}^{k}+\mu\left(P_{k} X-J_{k}\right) \\
& Y_{3}^{k+1}=Y_{3}^{k}+\mu\left(P_{k} X-S_{k}\right), Y_{4}^{k+1}=Y_{4}^{k}+\mu\left(H^{T}-X^{T} P_{k}^{T} C_{k}\right)
\end{aligned}
$$

Then, the optimization of DJ-RFDL can be described as:

\section{1) Fix others, update $J, S, F, W, V, D$ respectively:}

The updating rules of $J, S, F, W, V, D$ are the same as J-RFDL.

\section{2) Fix others, update the projection $P$ and $Q$ :}

By removing the terms irrelevant to $P$ and $Q$ from $\Omega$, we have

$$
\begin{aligned}
\min _{P, Q} \Omega(P, Q)=\operatorname{tr}\left(\alpha\left(V^{\mathrm{T}}-D P X\right)^{\mathrm{T}} Q\left(V^{\mathrm{T}}-D P X\right)\right)+\left\langle Y_{2}, P X-J\right\rangle \\
+\left\langle Y_{3}, P X-S\right\rangle+\left\langle Y_{4}, H^{\mathrm{T}}-X^{\mathrm{T}} P^{\mathrm{T}} C-E\right\rangle \\
+\frac{\mu}{2}\left(\|P X-J\|_{F}^{2}+\|P X-S\|_{F}^{2}+\left\|H^{\mathrm{T}}-X^{\mathrm{T}} P^{\mathrm{T}} C-E\right\|_{F}^{2}\right)
\end{aligned}
$$

By taking the derivative of $\Omega(P, Q)$ w.r.t. $P$ and setting it to zero, we can update the underlying projection $P_{k+1}$ as

$$
P_{k+1}=\left(2 \alpha D_{k+1}{ }^{T} Q_{k} D_{k+1}+2 \mu_{k+1} I+\mu C_{k} C_{k}^{T}\right)^{-1} Z_{k}\left(X X^{T}+\tau I\right)^{-1} .
$$

where $R_{k}=2 \alpha\left(X V_{k+1} Q_{k} D_{k+1}\right)^{T}, T_{k}=C_{k} Y_{4}^{T} X^{T}+\mu_{k} C_{k} H X^{T}-\mu_{k} C_{k} E^{T} X^{T}$ and $Z_{k}=\left(R_{k}-Y_{2}^{k} X^{T}-Y_{3}^{k} X^{T}+\mu_{k} J_{k} X^{T}+\mu_{k} S_{k} X^{T}+T_{k}\right)$. After $V, D$ and $P$ are obtained, we can update $Q$ by $\left(q_{i i}\right)_{k+1}=1 /\left(2\left\|\chi_{k+1}^{i}\right\|_{2}\right)$, where $\chi_{k+1}^{i}$ is $i$-th row of the matrix $V_{k+1}^{T}-D_{k+1} P_{k+1} X$.

\section{3) Fix others, update the classifier $\boldsymbol{C}$ and error $\boldsymbol{E}$ :}

We first describe how to update $C$ from the reduced problem:

$$
\min _{C} \beta\|C\|_{F}^{2}+\left\langle Y_{4}, H^{T}-X^{T} P^{T} C-E\right\rangle+\frac{\mu}{2}\left\|H^{T}-X^{T} P^{T} C-E\right\|_{F}^{2} .
$$

By taking the derivative w.r.t. $C$ and setting it to zero, we can update the classifier $C_{k+1}$ as

$$
\begin{aligned}
& \nabla_{k+1}=P_{k+1} X Y_{4}^{k} / \mu_{k}+P_{k+1} X H^{T}-P_{k+1} X E_{k} . \\
& C_{k+1}=\left(P_{k+1} X X^{T} P_{k+1}^{T}+2 \beta I / \mu_{k}\right)^{-1} \nabla_{k+1}
\end{aligned} .
$$

Then, the error matrix $E$ can be analogously inferred as

$$
E_{k+1}=\arg \min _{E} \frac{\beta}{\mu_{k}}\|E\|_{2,1}+\frac{1}{2}\left\|E-\left(H^{T}-X^{T} P_{k+1}{ }^{T} C_{k+1}+Y_{4}^{k} / \mu_{k}\right)\right\|_{F}^{2}
$$

The iterate $E_{k+1}$ can be obtained via the shrinkage operator as $E_{k+1}=\mho_{\beta / \mu}\left[\sum_{E}\right]$, where $\Sigma_{E}=\left(H^{T}-X^{T} P_{k+1}{ }^{T} C_{k+1}+Y_{4}^{k} / \mu_{k}\right)$. For complete presentation of the optimization of our DJ-RFDL, we summarize the procedures in Algorithm 2.

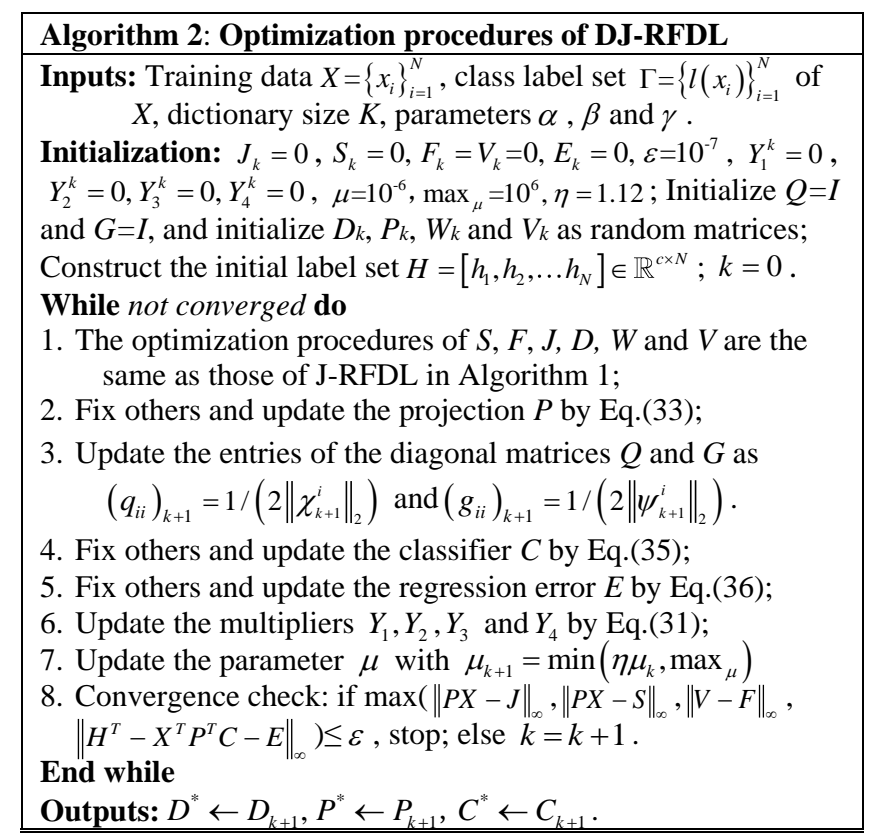

\section{Approach for Handling Outside New Data}

We mainly discuss our J-RFDL and DJ-RFDL for the inductive representation and classification of outside new data.

Learning the hybrid salient representations of new data. After $P^{*}$ is obtained and given a new sample $x_{\text {new }}, \mathrm{J}$-RFDL and DJ-RFDL obtain the hybrid salient coefficient vector as

$$
\aleph_{\text {new }}=P^{*} x_{\text {new }} \text {. }
$$

Classification of new data by DJ-RFDL. Since $P^{*}$ and $C^{*}$ are jointly obtained, we can involve the outside new data by them. Note that the classification process of DJ-RFDL is very efficient. For new sample $x_{\text {new }}$, its soft label vector $u_{\text {new }} \in \mathbb{R}^{c \times 1}$ and hard label $l\left(x_{\text {new }}\right)$ can be obtained as

$$
\begin{gathered}
l\left(x_{\text {new }}\right)=\arg \max _{i \leq c}\left(u_{\text {new }}\right)_{i}, \text { where } \\
u_{\text {new }}=C^{* T} \aleph_{\text {new }}, \aleph_{\text {new }}=P^{*} x_{\text {new }}
\end{gathered},
$$

where $\left(u_{\text {new }}\right)_{i}$ is the $i$-th entry of estimated soft vector $u_{\text {new }}$, i.e., the largest element in $\left(u_{\text {new }}\right)_{i}$ decides the label of $x_{\text {new }}$.

Classification of new data by J-RFDL. Since our J-RFDL fails to learn a classifier jointly, we propose to apply a similar process to obtain a linear classifier $C^{*}$ based on the computed hybrid salient representation $P^{*} X$ of data $X$ separately:

$$
\langle C, E\rangle=\arg \min _{C, E}\|E\|_{2,1}+\beta\|C\|_{F}^{2}, \text { s.t. } H^{T}=X^{T} P^{* T} C+E,
$$

which can be similarly solved as Eq.(36). After obtaining the linear classifier $C^{*}$, we can similarly obtain the soft label vector and hard label of each new data $x_{\text {new }}$ by Eq. (38).

\section{DisCUSSION: RELATIONSHIP ANALYSIS}

We will describe some important relations to our algorithm. 


\section{A. Connection between $C F$ and J-RFDL}

Recalling the objective function of J-RFDL in Eq.(8), suppose that $\alpha=0$ and $\gamma=0$, Eq.(8) can be transformed into

$$
\min _{W, V}\left\|X^{T}-V W^{T} X^{T}\right\|_{2,1}=\operatorname{tr}\left(\left(X^{T}-V W^{T} X^{T}\right)^{T} G\left(X^{T}-V W^{T} X^{T}\right)\right) .
$$

If we fix the diagonal matrix $G$ to be identity matrix, Eq.(40) becomes the following Frobenius-norm based one:

$$
\min _{W, V} \operatorname{tr}\left(\left(X^{T}-V W^{T} X^{T}\right)^{T}\left(X^{T}-V W^{T} X^{T}\right)\right)=\left\|X-X W V^{T}\right\|_{F}^{2},
$$

which is just the problem of CF. In other words, CF is a special example of our J-RFDL. But by replacing the $L_{2,1}$-norm with Frobenius-norm, the metric will lose the robust properties.

\section{B. Connection between DPL and J-RFDL}

We then show the relationship between DPL and J-RFDL. For the objective function of our J-RFDL in Eq.(8), if we constrain $\gamma=0$, the problem of Eq.(8) can be reduced into

$$
\min _{D, P, W, V}\left\|X^{T}-V W^{T} X^{T}\right\|_{2,1}+\alpha\left(\left\|V^{\mathrm{T}}-D P X\right\|_{2,1}+\|V\|_{1}\right) \text {, s.t. } W, V \geq 0 .
$$

Suppose the ideal case that the reconstructed data $V W^{T} X^{T}$ is equal to the original data $X^{T}$ is satisfied, i.e., the reconstruction error $\left\|X^{T}-V W^{T} X^{T}\right\|_{2,1}$ is zero, by removing the $L_{1}$-norm sparse constraint on $V$ and adding the constraint $\left\|d_{i}\right\|_{2}^{2} \leq 1$, we have

$$
\min _{D, P, Q}\left\|V^{\mathrm{T}}-D P X\right\|_{2,1}=\operatorname{tr}\left(\left(V^{\mathrm{T}}-D P X\right)^{T} Q\left(V^{\mathrm{T}}-D P X\right)\right) \text {, s.t. }\left\|d_{i}\right\|_{2}^{2} \leq 1 .
$$

If we also fix $Q$ to be the identity matrix, the above problem becomes the Frobenius-norm based one. Note that although our J-RFDL also involves a projection to approximate the codes by embedding similarly as DPL, they are different in three aspects. First, DPL is a supervised method and needs label information of all training samples, so its performance may be restricted due to limited number of labeled samples in practice, while J-RFDL is essentially an unsupervised algorithm. Second, DPL is a structured DL method and directly uses an analysis DL strategy for approximating the coefficients without using any constraint on the coefficients, while J-RFDL can obtain the hybrid salient low-rank and sparse coefficients clearly. Third, DPL performs in the original input space, while J-RFDL conducts the hybrid representation in factorized compressed space and moreover the factorization process can also remove the noise, which can further improve the performance. By using the Frobenius-norm to replace the sparse and robust $L_{2,1}$-norm, DPL will lose the robust property against noise and outliers in given data, and the reconstruction may be inaccurate in reality.

\section{Simulation Results And Analysis}

We conduct the simulations to evaluate the effectiveness of our J-RFDL and DJ-RFDL, along with illustrating the comparison results with the other related representation and classification techniques. We perform all the simulations on a PC with Intel (R) Core (TM) i7-7700 CPU @ 3.6 GHz 8G.

\section{A. Baseline and Setting}

We mainly evaluate J-RFDL for unsupervised representation and evaluate DJ-RFDL for classification. The representation result of our J-RFDL is mainly compared with those of closely related low-rank and/or sparse coding algorithms, i.e., KSVD [20], IRPCA [14], LatLRR [15], rLRR [16] and I-LSPFC [18]. For classification, we compare our J-RFDL and DJ-RFDL with the unsupervised KSVD, IRPCA, LatLRR, I-LSPFC and rLRR, as well as the supervised D-KSVD [25], LC-KSVD1 [27], LC-KSVD2 [27], Discriminative I-LSPFC (D-LSPFC) [18], DLSI[21], ADDL [22], DPL [17] and LRSDL [52]. Note that IRPCA, LatLRR, rLRR and I-LSPFC are unsupervised models and cannot classify new data directly, so we employ the same classifier training and classification process by Eqs.(38-39) as our J-RFDL for them for the fair comparison. For KSVD, we similarly compute a classifier as the classifier training process separately as the LC-KSVD1 algorithm.

Four kinds of images, including face images, object images, handwriting digital images and natural scene images, are tested. Details of used databases are shown in Table I. As a common practice, each face image is resized into $32 \times 32$ pixels, which corresponds to a 1024-D vector. For classification, we split each dataset into a training set and a test set, where the training set is used for representation and classifier learning, and the test set is used for evaluation. For unsupervised models, the training set has no supervised prior, while all the training samples are labeled for supervised methods. The classification accuracy of each method is finally obtained by comparing the predicted labels with the ground-truth labels provided by data corpus.

Table I: Descriptions of Used Image Datasets.

\begin{tabular}{c|c|c|c}
\hline \hline Dataset Name & \# Samples & \# Dim & \# Classes \\
\hline COIL20 object [43] & 1440 & 1024 & 20 \\
UMIST face [49] & 1012 & 1024 & 20 \\
CMU PIE face [44] & 11554 & 1024 & 68 \\
MIT CBCL face [57] & 3240 & 1024 & 10 \\
USPS digits [23] & 9298 & 256 & 10 \\
MNIST digits [58] & 70000 & 784 & 10 \\
Optical digits (OHD) [45] & 5620 & 64 & 10 \\
Caltech101 objects [62] & 9144 & 4096 & 101 \\
Fifteen natural scenes [63] & 4485 & 4096 & 15 \\
\hline \hline
\end{tabular}

\section{B. Investigation of Parameters}

We analyze the parameter sensitivity of J-RFDL and DJ-RFDL. Since parameter selection is still an open issue, a heuristic way is used to select the most important ones. The COIL20 object database [43] is evaluated. For our J-RFDL, we can explore the effects of parameters $\alpha$ and $\gamma$ on the classification results by grid search. To see the effects of parameters, we randomly select 20 images from each subject for training and test on the rest. We average the results over 10 random splits of training and test samples with varied parameters from the candidate set $\left\{10^{-9}, \ldots, 10^{1}\right\}$. The results are shown in Fig.2. We find that J-RFDL performs well for a wide range of parameter settings. In this paper, we simply set $\alpha=1$ and $\gamma=10^{-5}$ for J-RFDL.

DJ-RFDL includes three model parameters, so we fix one of them and explore the effects of other two on the result by grid search. To see the effects of different parameters, we first fix $\alpha=10^{-5}$ and tune $\beta$ and $\gamma$ by grid search from the candidate set $\left[10^{-9}, 10^{-8}, \ldots, 10^{1}\right]$ in Fig.3(a), from which we can find that DJ-RFDL with $\beta \geq 10^{-5}$ and $\gamma \leq 10^{-2}$ can deliver good and stable results. We then fix $\gamma=10^{-3}$ to explore the effects of $\beta$ and $\alpha$ in Fig.3(b), from which we can see that DJ-RFDL with $\beta \geq 10^{-5}$ and $\alpha \leq 10^{-1}$ can deliver good results. Finally, we fix $\beta=10^{-3}$ and explore the effects of $\gamma$ and $\alpha$ in Fig.3(c), and we can observe that DJ-RFDL with each $\gamma$ and $\alpha \leq 10^{-1}$ can deliver good and 
stable results. Note that similar findings can be obtained from other dataset, so we simply select the parameters from the range of $\alpha \leq 10^{-1}, \beta \geq 10^{-5}$ and $\gamma \leq 10^{-2}$ for DJ-RFDL in this paper.

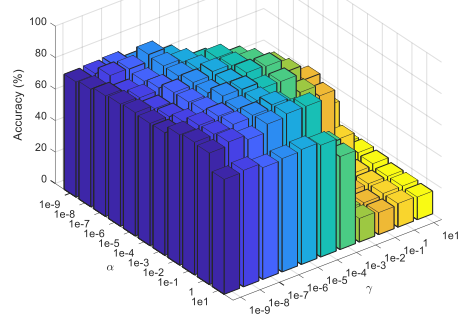

Fig. 2: Parameter sensitivity analysis of J-RFDL on COIL20 database.

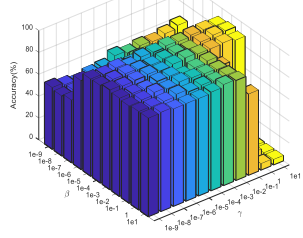

(a)

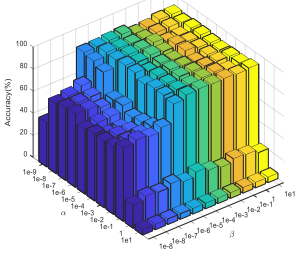

(b)

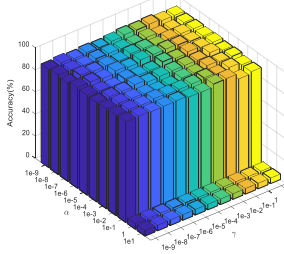

(c)
Fig. 3: Parameter sensitivity analysis of our DJ-RFDL on COIL20, where (a) fix $\alpha$ to tune $\beta$ and $\gamma$ using grid search; (b) fix $\gamma$ to tune $\beta$ and $\alpha$ using grid search; (c) fix $\beta$ to tune $\gamma$ and $\alpha$ using grid search.

Table II: Classification results of J-RFDL under different parameter settings on three databases.

\begin{tabular}{c|c|c|c}
\hline Parameter settings & MIT CBCL & COIL20 & USPS \\
\hline$\alpha \neq 0, \gamma \neq 0$ & $\mathbf{9 6 . 1 2}$ & $\mathbf{8 4 . 3 3}$ & $\mathbf{8 2 . 3 8}$ \\
\hline$\alpha=0, \gamma \neq 0$ & 8.69 & 4.93 & 11.75 \\
\hline$\alpha \neq 0, \gamma=0$ & 91.96 & 82.88 & 63.19 \\
\hline
\end{tabular}

Table III: Classification results of DJ-RFDL under different parameter settings on three databases.

\begin{tabular}{c|c|c|c}
\hline Parameter settings & MIT CBCL & COIL20 & USPS \\
\hline$\alpha \neq 0, \beta \neq 0, \quad \gamma \neq 0$ & $\mathbf{9 8 . 3 1}$ & $\mathbf{9 4 . 0 5}$ & $\mathbf{8 2 . 2 0}$ \\
\hline$\alpha=0, \beta \neq 0, \quad \gamma \neq 0$ & 40.24 & 33.12 & 52.71 \\
\hline$\alpha \neq 0, \beta=0, \quad \gamma \neq 0$ & 91.68 & 66.34 & 57.07 \\
\hline$\alpha \neq 0, \beta \neq 0, \quad \gamma=0$ & 92.21 & 90.92 & 19.37 \\
\hline
\end{tabular}

In addition to the above parameter analysis, we also explore the effects and contributions of the involved terms associated with the model parameters in the objective functions of J-RFDL and DJ-RFDL by setting the parameters to 0 , respectively. In this study, MIT CBCL, COIL20 and USPS databases are used. MIT CBCL database has images of 10 persons, and each person has 324 images. The head models are generated by fitting a morphable model to the high-resolution training images. USPS database has 9298 handwritten digits ('0'-'9') of $16 \times 16$ pixels [23]. In this paper, we choose 300 samples from each digit and each digit is represented by a 256-dimensional vector. In this experiment, we select 4, 20 and 40 per class from these datasets respectively for training. The results of J-RFDL and DJ-RFDL are shown in Tables II and III. We can find that when we set $\alpha, \beta$ or $\gamma$ to 0 , the classification results are all decreased. More specifically, when $\alpha=0$ in J-RFDL and DJ-RFDL, i.e., the reconstruction term of DL is removed, the result is the lowest. That is, this reconstruction term is more important. When $\gamma=0$ in J-RFDL and DJ-RFDL, i.e., the hybrid salient representation coefficients are not be obtained any more, the classification result is also decreased. If $\beta=0$ in DJ-RFDL, i.e., the linear classifier $C$ is not optimized jointly, the classification result is still decreased. This implies that minimizing the classification error based on hybrid salient coefficients at the same time is needed. Hence, the involved several terms in the problems of our methods are all important to improve the performance.

It should be noted that the model parameter(s) of the other compared algorithms are also carefully chosen from the same candidate set as our methods for the fair comparison, and the presented results of other compared algorithms below are also based on the chosen best parameters from candidate set. But due to the page limitation, we will not describe the parameter selection results of other compared algorithms, because this is beyond the main scope of this paper.

\section{Comparison of Actual Computation Time}

For clear observation, Fig.4 shows the averaged training and testing time separately on three databases, i.e., UMIST [49], CMU PIE [44] and COIL20 [43]. Each subject of UMIST is described in a range of poses from profile to frontal views. Following the common procedures over CMU PIE, 170 near frontal face images per person are used, containing five near frontal poses (C05, C07, C09, C27, and C29), and all images have different illuminations, lighting, and expressions.

For each database, we randomly select 20 simples from each class for training and test on the rest. We can see from Figs.4(a) and (c) that: (1) DPL and ADDL are slightly faster than other methods, and our J-RFDL and DJ-RFDL are faster than the KSVD, D-KSVD, LC-KSVD1 and LC-KSVD2; (2) J-RFDL, DJ-RFDL, DPL and ADDL spend relatively less time in testing, compared with KSVD, D-KSVD and LC-KSVD. For the result in Fig.4 (b) on a larger face database, KSVD, D-KSVD and LC-KSVD spend much more time in testing than others.

Besides, we also prepare a simulation to evaluate the effects of using the whole dataset instead of selected number of images on the time consumption. UMIST, CMU PIE and COIL20 are also used. That is, all the samples are used for training and we report the training time in Fig.5. For UMIST, the dictionary size is set to 450. For CMU PIE, we use the principal component analysis (PCA) to reduce the dimension by preserving $99 \%$ energy. We can find that DPL and ADDL are still faster than other methods, and J-RFDL and DJ-RFDL deliver comparable or even less running time than KSVD, D-KSVD, LC-KSVD1 and LC-KSVD2 algorithms in most cases.

\section{Convergence Analysis Results}

We present some convergence results of our proposed J-RFDL and DJ-RFDL. In this study, four image databases, i.e., COIL20, UMIST, MIT CBCL and USPS, are used. For each database, we select 10 images per class for training and the averaged convergence results over 10 splits are shown in Fig.6. We can find that the convergence errors of our J-RFDL and DJ-RFDL are non-increasing and usually converge to a very small value. Moreover, J-RFDL and DJ-RFDL usually converge with the number of iterations ranging from 20 to 60 in most cases.

\section{E. Face Recognition}

We evaluate J-RFDL and DJ-RFDL for face recognition on two public real databases, i.e., CMU PIE [44] and MIT CBCL [57]. Some image examples are illustrated in Fig.7. The number of atoms is set as its maximum for each method. 


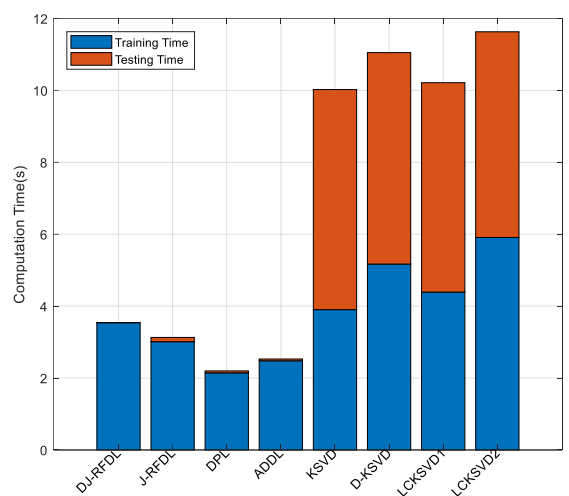

(a) UMIST

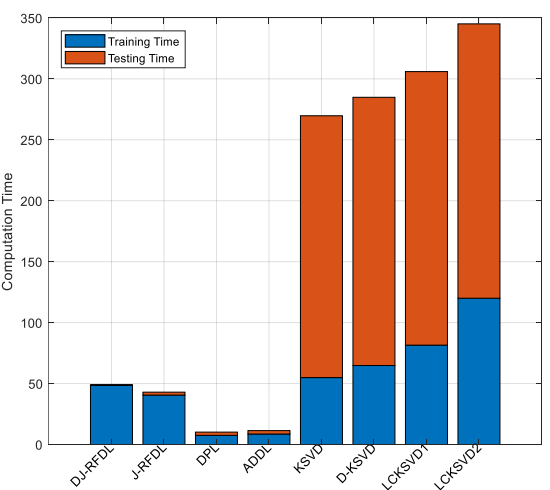

(b) CMU PIE

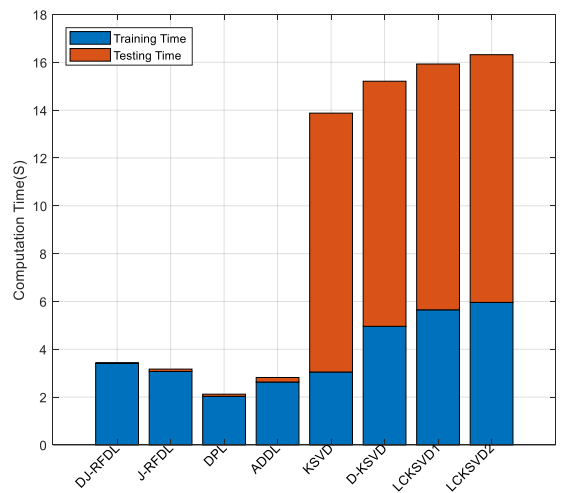

(c) COIL20

Fig.4: Comparison of the training and test time (in seconds) consumption on three image databases.

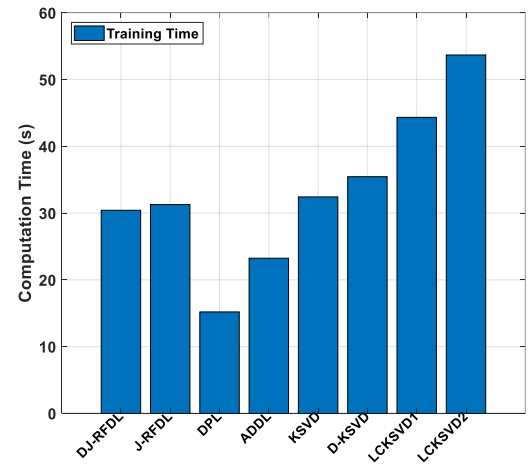

(a) UMIST

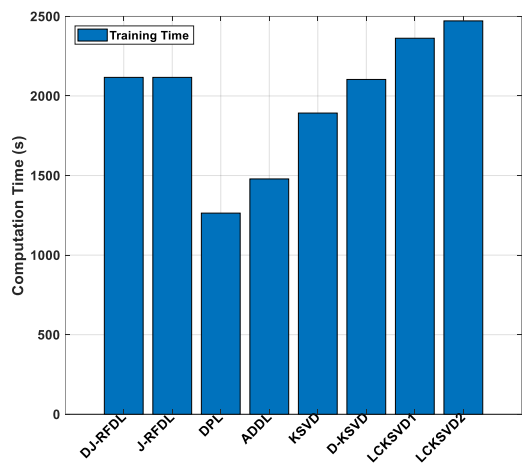

(b) CMU PIE

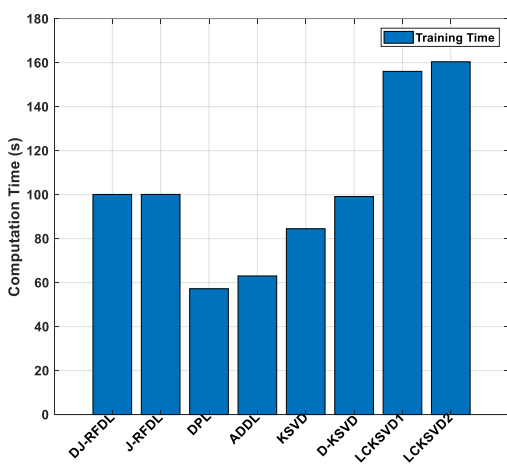

(c) COIL20

Fig.5: Comparison of the training time consumption(in seconds) on three image databases, where all samples are used for training.

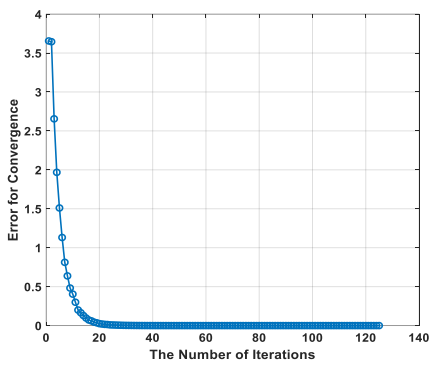

(a1) COIL20

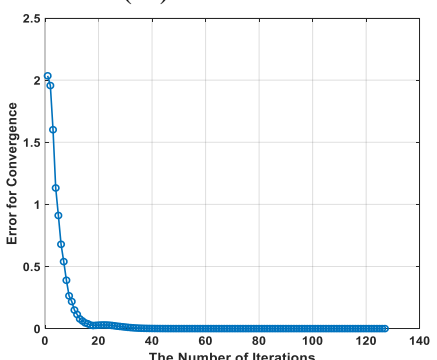

(a2) COIL20

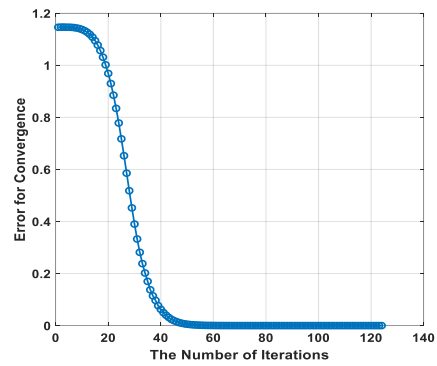

(b1) UMIST

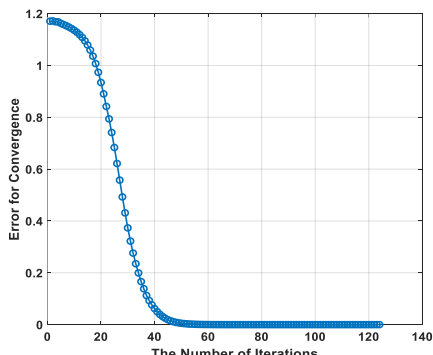

(b2) UMIST

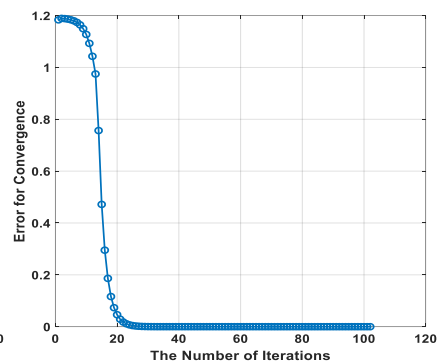

(c1) MIT CBCL

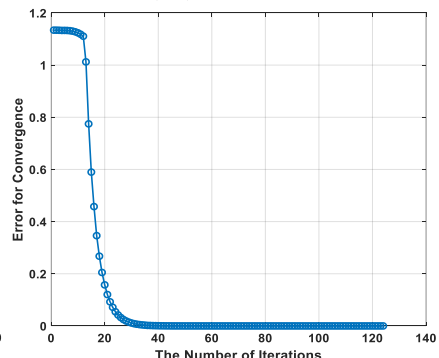

(c2) MIT CBCL

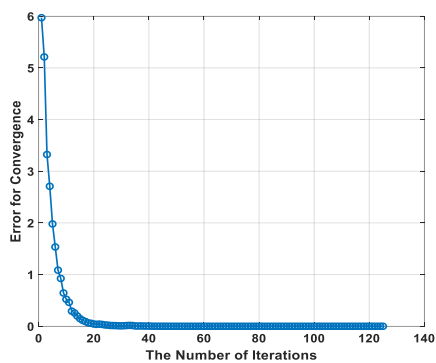

(d1) USPS

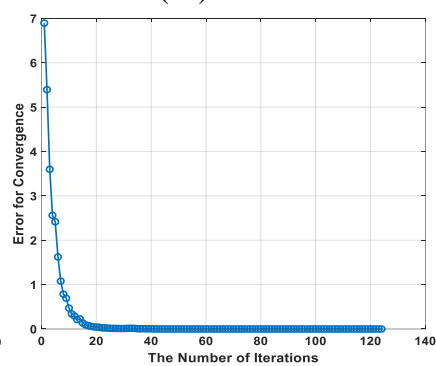

(d2) USPS

Fig.6: The convergence results of our J-RFDL (a1-d1) and DJ-RFDL (a2-d2) on four real-world image databases.

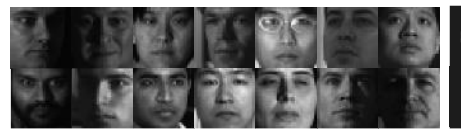

(a) CMU PIE

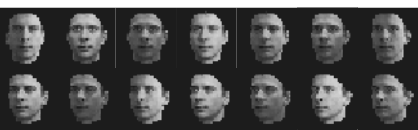

(b) MIT CBCL

Fig. 7: Image examples of the evaluated face databases.

Recognition results on CMU PIE. We mainly compare the results of J-RFDL and DJ-RFDL with those of unsupervised

KSVD, LatLRR, rLRR, IRPCA, I-LSPFC, and discriminative D-KSVD, DLSI, LC-KSVD, D-LSPFC, DPL and ADDL in this study. Table IV shows the results of each method, where we fix the number $(f)$ of training faces per person and average the results over 10 random splits of training/testing images. We report the mean accuracy (\%) with the standard deviation (STD) and highest accuracy (\%). Note that we show the results by two groups, i.e., unsupervised and supervised ones, and highlight 
the best record in each group in bold. For unsupervised group, we compare our J-RFDL with KSVD, LatLRR, rLRR, IRPCA, I-LSPFC. $\alpha=10^{-4}, \beta=10^{-4}$ and $\gamma=10^{-4}$ are used in DJ-RFDL and we have the following observations. (1) For unsupervised methods, J-RFDL usually obtains better results than compared methods. I-LSPFC and rLRR also work well by obtaining the comparable results to J-RFDL. The superiority of rLRR over LatLRR can be attributed to preserving the manifold structures of features. (2) For supervised group, DJ-RFDL obtains higher accuracies across all numbers of training samples.
In addition, we also compare the average recognition rates by varying the dictionary size $K$. The training set is formed by randomly choosing 30 images per person. $K=340,680,1020$, 1360, 1700, and 2040 are tested, and the results are illustrated in Fig. 8(a). We see that the rates of each method are increasing along with the increasing numbers of dictionary atoms, and more importantly DJ-RFDL can outperform all its competitors by delivering the enhanced performance. ADDL also performs well by delivering enhanced recognition performance.

Table IV: Performance comparison of the algorithms on the CMU PIE face database.

\begin{tabular}{|c|c|c|c|c|c|c|c|c|}
\hline \multirow{2}{*}{ Setting } & \multicolumn{2}{|c|}{ CMU PIE (3 train) } & \multicolumn{2}{c|}{ CMU PIE (5 train) } & \multicolumn{2}{c|}{ CMU PIE (7 train) } & \multicolumn{2}{c|}{ CMU PIE (9 train) } \\
\cline { 2 - 9 } Method & Mean \pm STD & Best & Mean \pm STD & Best & Mean \pm STD & Best & Mean \pm STD & Best \\
\hline \hline IRPCA & $49.15 \pm 1.82$ & 52.51 & $58.61 \pm 1.69$ & 61.29 & $64.59 \pm 1.35$ & 66.55 & $73.48 \pm 1.02$ & 75.89 \\
\hline LatLRR & $53.95 \pm 1.08$ & 56.21 & $60.41 \pm 0.93$ & 61.26 & $70.69 \pm 0.99$ & 72.04 & $76.42 \pm 1.29$ & 78.95 \\
\hline rLRR & $54.13 \pm 2.21$ & $\mathbf{5 6 . 9 6}$ & $64.36 \pm 1.32$ & 66.42 & $74.38 \pm 0.63$ & 75.04 & $78.56 \pm 0.89$ & $\mathbf{8 0 . 5 2}$ \\
\hline KSVD & $48.22 \pm 1.73$ & 52.04 & $55.31 \pm 1.27$ & 57.33 & $66.34 \pm 0.62$ & 68.21 & $75.59 \pm 0.33$ & 77.12 \\
\hline I-LSPFC & $51.82 \pm 0.38$ & 52.10 & $63.21 \pm 1.17$ & 65.15 & $70.11 \pm 1.15$ & 72.28 & $74.54 \pm 0.70$ & 75.26 \\
\hline J-RFDL & $\mathbf{5 5 . 4 1 \pm 0 . 6 5}$ & 56.15 & $\mathbf{6 8 . 5 5} \pm \mathbf{1 . 9 1}$ & $\mathbf{7 1 . 1 9}$ & $\mathbf{7 4 . 7 1} \pm \mathbf{0 . 8 4}$ & $\mathbf{7 5 . 6 8}$ & $\mathbf{7 9 . 0 6} \pm \mathbf{0 . 7 3}$ & 79.78 \\
\hline \hline DLSI & $33.10 \pm 1.47$ & 34.99 & $49.13 \pm 1.19$ & 50.71 & $64.13 \pm 1.03$ & 65.77 & $74.09 \pm 1.16$ & 75.67 \\
\hline ADDL & $57.59 \pm 1.42$ & 59.11 & $68.84 \pm 1.35$ & 70.15 & $76.49 \pm 0.56$ & 77.33 & $80.82 \pm 0.66$ & 81.55 \\
\hline LC-KSVD1 & $52.38 \pm 1.35$ & 54.04 & $63.75 \pm 1.43$ & 66.27 & $70.33 \pm 0.52$ & 70.09 & $76.92 \pm 0.72$ & 77.66 \\
\hline LC-KSVD2 & $55.12 \pm 1.24$ & 56.98 & $67.94 \pm 1.31$ & 68.26 & $73.33 \pm 0.72$ & 73.83 & $77.62 \pm 0.48$ & 78.53 \\
\hline DPL & $57.04 \pm 1.65$ & 59.03 & $70.34 \pm 1.39$ & 71.98 & $77.13 \pm 2.33$ & 79.16 & $82.41 \pm 0.67$ & 83.69 \\
\hline D-KSVD & $50.17 \pm 3.69$ & 54.22 & $59.35 \pm 3.92$ & 64.54 & $67.82 \pm 0.89$ & 69.36 & $76.51 \pm 1.10$ & 77.76 \\
\hline D-LSPFC & $58.05 \pm 1.25$ & 60.48 & $68.39 \pm 0.65$ & 69.14 & $76.89 \pm 0.81$ & 78.36 & $81.21 \pm 0.19$ & 82.07 \\
\hline LRSDL & $55.86 \pm 2.37$ & 58.42 & $67.18 \pm 1.21$ & 70.35 & $77.22 \pm 0.91$ & 89.27 & $79.60 \pm 1.23$ & 82.47 \\
\hline DJ-RFDL & $\mathbf{6 1 . 0 8} \pm \mathbf{1 . 4 1}$ & $\mathbf{6 3 . 7 3}$ & $\mathbf{7 3 . 0 8} \pm \mathbf{0 . 8 6}$ & $\mathbf{7 4 . 2 9}$ & $\mathbf{8 0 . 7 5} \pm \mathbf{0 . 5 8}$ & $\mathbf{8 1 . 8 1}$ & $\mathbf{8 4 . 4 0 \pm 0 . 9 1}$ & $\mathbf{8 5 . 7 2}$ \\
\hline \hline
\end{tabular}

Table V: Performance comparison of the algorithms on the MIT CBCL face database.

\begin{tabular}{|c|c|c|c|c|c|c|c|c|}
\hline \multirow{2}{*}{ Setting } & \multicolumn{2}{|c|}{ MIT CBCL (1 train) } & \multicolumn{2}{c|}{ MIT CBCL (2 train) } & \multicolumn{2}{c|}{ MIT CBCL (3 train) } & \multicolumn{2}{c|}{ MIT CBCL (4 train) } \\
\cline { 2 - 9 } & Mean \pm STD & Best & Mean \pm STD & Best & Mean \pm STD & Best & Mean \pm STD & Best \\
\hline \hline IRPCA & $62.00 \pm 3.50$ & $\mathbf{6 7 . 4 6}$ & $82.18 \pm 4.79$ & 88.59 & $92.35 \pm 3.13$ & 96.79 & $95.25 \pm 3.75$ & 99.28 \\
\hline LatLRR & $51.07 \pm 2.09$ & 55.29 & $63.99 \pm 4.70$ & 68.94 & $77.16 \pm 3.42$ & 81.45 & $80.32 \pm 3.30$ & 85.52 \\
\hline rLRR & $60.71 \pm 3.57$ & 65.29 & $70.78 \pm 6.30$ & 84.65 & $85.29 \pm 4.16$ & 90.69 & $84.51 \pm 3.07$ & 88.72 \\
\hline KSVD & $55.41 \pm 1.23$ & 57.36 & $62.57 \pm 2.31$ & 64.39 & $86.89 \pm 6.17$ & 91.90 & $93.15 \pm 2.42$ & 95.90 \\
\hline I-LSPFC & $57.71 \pm 3.68$ & 66.59 & $83.70 \pm 4.08$ & $\mathbf{8 9 . 5 3}$ & $92.50 \pm 3.60$ & 96.70 & $95.95 \pm 1.41$ & 97.52 \\
\hline J-RFDL & $\mathbf{6 3 . 8 1} \pm \mathbf{3 . 2 7}$ & 66.84 & $\mathbf{8 5 . 9 1} \pm \mathbf{2 . 3 3}$ & 87.33 & $\mathbf{9 3 . 6 5} \pm \mathbf{3 . 1 3}$ & $\mathbf{9 7 . 0 7}$ & $\mathbf{9 6 . 1 2} \pm \mathbf{2 . 3 0}$ & $\mathbf{9 9 . 5 9}$ \\
\hline \hline DLSI & $60.44 \pm 3.14$ & 65.32 & $74.72 \pm 3.29$ & 77.08 & $89.31 \pm 3.37$ & 93.42 & $89.50 \pm 4.24$ & 93.49 \\
\hline ADDL & $62.50 \pm 2.62$ & 65.83 & $84.42 \pm 5.71$ & 92.12 & $93.23 \pm 2.74$ & 96.91 & $97.25 \pm 1.62$ & 99.42 \\
\hline LC-KSVD1 & $59.27 \pm 3.67$ & 63.23 & $80.17 \pm 4.33$ & 85.76 & $90.13 \pm 3.47$ & 95.52 & $93.33 \pm 3.34$ & 95.43 \\
\hline LC-KSVD2 & $61.83 \pm 2.45$ & 64.31 & $83.35 \pm 5.63$ & 89.88 & $92.11 \pm 3.67$ & 96.32 & $94.15 \pm 3.05$ & 96.37 \\
\hline DPL & $60.81 \pm 1.81$ & 62.47 & $85.25 \pm 4.73$ & 91.42 & $93.81 \pm 1.37$ & 96.14 & $96.53 \pm 1.62$ & 99.50 \\
\hline D-KSVD & $58.23 \pm 3.23$ & 63.21 & $84.15 \pm 6.16$ & 91.43 & $91.69 \pm 3.78$ & 94.17 & $95.62 \pm 1.61$ & 97.19 \\
\hline D-LSPFC & $62.03 \pm 2.81$ & 67.33 & $85.66 \pm 4.26$ & 92.31 & $93.91 \pm 3.38$ & $\mathbf{9 8 . 0 1}$ & $96.47 \pm 1.48$ & 98.96 \\
\hline LRSDL & $59.05 \pm 2.42$ & 64.19 & $83.61 \pm 3.96$ & 89.57 & $93.42 \pm 2.82$ & 97.68 & $97.41 \pm 1.28$ & 98.49 \\
\hline DJ-RFDL & $\mathbf{6 4 . 3 6} \pm \mathbf{3 . 1 9}$ & $\mathbf{7 0 . 7 7}$ & $\mathbf{8 6 . 8 3} \pm \mathbf{5 . 3 2}$ & $\mathbf{9 5 . 5 9}$ & $\mathbf{9 6 . 0 1} \pm \mathbf{1 . 5 1}$ & 97.76 & $\mathbf{9 8 . 3 1 \pm 1 . 4 5}$ & $\mathbf{1 0 0}$ \\
\hline \hline
\end{tabular}

Recognition results on MIT CBCL. We test each method for recognizing the faces of MIT CBCL. Table V describes the result of each method, where we fix the number $(f)$ of training faces of each person (i.e., $f=1,2,3,4)$ and average the results over 10 random splits. We show the mean and highest accuracy (\%) for each method, and also divide the results into two groups according to the unsupervised and supervised methods. We find that J-RFDL and DJ-RFDL deliver comparable or better results than the other algorithms in each group. $\alpha=10^{-4}, \beta=10^{-4}$ and $\gamma=10^{-4}$ are applied for our DJ-RFDL for simulations.

Additionally, we randomly choose 6 samples from each class as training set and evaluate each DL method with varying size
$K$ of dictionary, i.e., $K=20,30,40,50,60$, in Fig. 8(b) that indicates that our DJ-RFDL maintains a higher recognition rate than other methods even when the dictionary size is relatively small. ADDL also performs well by delivering better results.

\section{F. Handwriting Recognition}

We also evaluate each method for handwriting recognition on three public handwritten databases, i.e., USPS [23] and OHD [45] and MNIST, and some image examples are shown in Fig.9.

Recognition results on USPS. We first evaluate each model to recognize the handwritten digits of USPS. Table VI shows the recognition result of each approach with varying numbers 


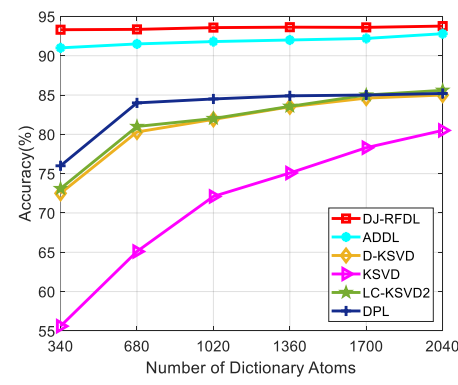

(a)

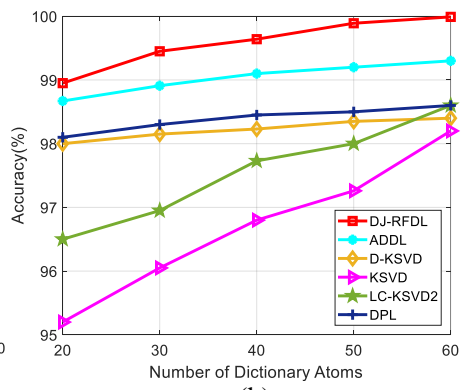

(b)

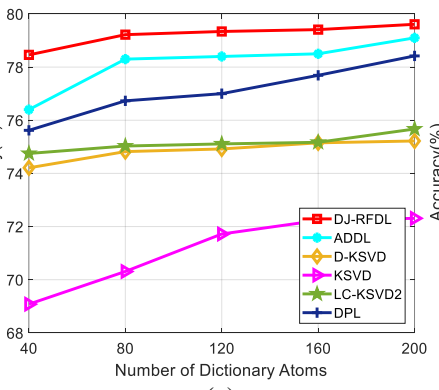

(c)

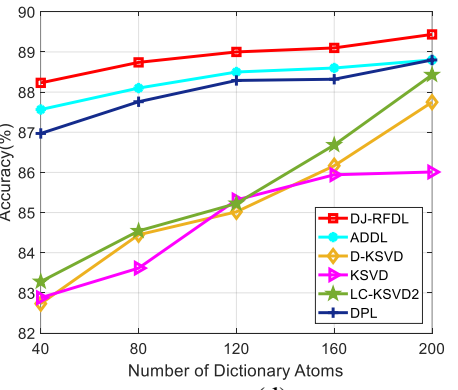

(d)

Fig. 8: Classificaton performance with varying dictionary sizes on (a) CMU PIE, (b) MIT CBCL, (c) USPS and (d) OHD databases

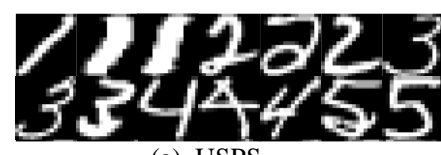

(a) USPS

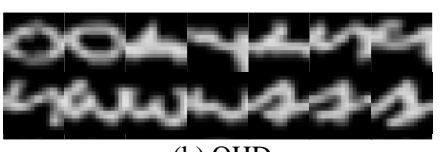

(b) OHD

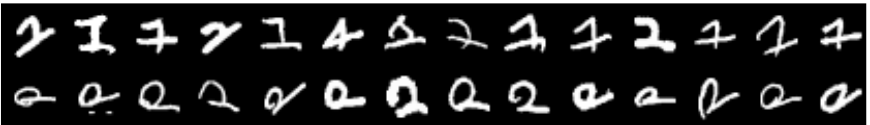

(c) MNIST

Fig. 9: Image examples of the evaluated handwritten databases.

of training data, i.e., 10, 20, 30 and 40 images per class, and test on the rest. The averaged results are shown in Table VI, where we report the mean accuracy with STD and highest record, and also show the results using two groups. $\alpha=10^{-1}, \beta=10^{-3}$ and $\gamma=10^{-6}$ are used for our DJ-RFDL. We can find that: (1) for unsupervised methods, J-RFDL obtains the highest accuracy on average, especially when the number of training data is small. (2) For discriminative group, DJ-RFDL outperforms the other

algorithms across all the training numbers, especially when the number of training samples is small. ADDL also works well.

To evaluate DJ-RFDL with varying dictionary sizes $K=40$, $80,120,160,200$. We randomly select 20 images per class to form the training set and test on the rest. The results under varying dictionary sizes are shown in Fig. 8(c). We can see that our methods can obtain higher accuracies than other methods.

Recognition results on OHD database. This database has handwritten digits (' 0 '-' 9 ') of $8 \times 8$ pixels where each element is an integer in the range from 0 to 16 . Table VII shows the result of each method with varying numbers of training data, i.e., 3, 6, 9 and 12 images per class. The results are averaged over 10 random splits of training and test digits. $\alpha=10^{-5}, \beta=10^{-5}$ and $\gamma=10^{-7}$ are used for DJ-RFDL. We find that for unsupervised and discriminative group, our methods can obtain better results than other methods, and the difference between ours and other models is larger when the number of training samples is small.

TABLE VI: Performance comparison of each algorithm on the USPS database.

\begin{tabular}{|c|c|c|c|c|c|c|c|c|}
\hline \multirow{2}{*}{ Setting } & \multicolumn{2}{|c|}{ USPS (10 train) } & \multicolumn{2}{c|}{ USPS (20 train) } & \multicolumn{2}{c|}{ USPS (30 train) } & \multicolumn{2}{c|}{ USPS (40 train) } \\
\cline { 2 - 9 } Method & Mean \pm STD & Best & Mean \pm STD & Best & Mean \pm STD & Best & Mean \pm STD & Best \\
\hline \hline IRPCA & $62.52 \pm 2.16$ & 65.17 & $70.47 \pm 2.48$ & 73.96 & $72.88 \pm 1.82$ & 76.81 & $76.75 \pm 1.61$ & 79.73 \\
\hline LatLRR & $63.18 \pm 2.18$ & 66.17 & $71.04 \pm 1.87$ & 73.08 & $73.31 \pm 0.84$ & 74.52 & $75.22 \pm 0.94$ & 76.58 \\
\hline rLRR & $65.80 \pm 2.09$ & $\mathbf{6 9 . 5 2}$ & $72.17 \pm 2.31$ & $\mathbf{7 5 . 1 1}$ & $74.23 \pm 2.06$ & 77.11 & $76.83 \pm 0.91$ & 79.73 \\
\hline KSVD & $64.62 \pm 1.47$ & 67.14 & $72.21 \pm 1.69$ & 72.94 & $77.55 \pm 1.59$ & 80.22 & $80.25 \pm 0.71$ & 81.62 \\
\hline I-LSPFC & $63.87 \pm 1.97$ & 66.97 & $71.39 \pm 2.28$ & 74.29 & $76.00 \pm 1.57$ & 77.85 & $78.37 \pm 0.91$ & 80.19 \\
\hline J-RFDL & $\mathbf{6 6 . 0 0} \pm \mathbf{2 . 0 5}$ & 68.83 & $\mathbf{7 4 . 0 6} \pm \mathbf{0 . 8 4}$ & 74.89 & $\mathbf{7 8 . 3 8} \pm \mathbf{1 . 1 3}$ & $\mathbf{8 0 . 2 6}$ & $\mathbf{8 2 . 3 8} \pm \mathbf{0 . 3 6}$ & $\mathbf{8 3 . 0 8}$ \\
\hline \hline DLSI & $67.47 \pm 1.78$ & 70.12 & $74.69 \pm 1.29$ & 77.61 & $78.59 \pm 1.27$ & 80.91 & $82.42 \pm 1.12$ & 83.47 \\
\hline ADDL & $69.71 \pm 1.72$ & 72.52 & $78.35 \pm 1.59$ & 80.21 & $80.79 \pm 0.63$ & 81.82 & $83.06 \pm 1.14$ & 84.92 \\
\hline LC-KSVD1 & $63.07 \pm 0.41$ & 64.09 & $74.88 \pm 0.73$ & 76.00 & $77.83 \pm 0.61$ & 78.52 & $78.83 \pm 2.16$ & 80.96 \\
\hline LC-KSVD2 & $67.90 \pm 1.28$ & 68.75 & $75.28 \pm 1.99$ & 77.57 & $79.42 \pm 1.17$ & 80.04 & $81.79 \pm 0.56$ & 82.69 \\
\hline DPL & $68.75 \pm 1.93$ & 71.45 & $77.43 \pm 1.06$ & 78.68 & $80.37 \pm 0.59$ & 81.26 & $82.75 \pm 0.72$ & 83.77 \\
\hline D-KSVD & $65.58 \pm 1.82$ & 68.10 & $75.05 \pm 1.71$ & 76.46 & $78.87 \pm 0.58$ & 79.37 & $80.91 \pm 0.68$ & 81.65 \\
\hline D-LSPFC & $66.58 \pm 1.63$ & 69.24 & $74.17 \pm 2.21$ & 76.89 & $78.69 \pm 2.94$ & 82.15 & $81.74 \pm 2.41$ & 84.19 \\
\hline LRSDL & $69.14 \pm 1.67$ & 72.58 & $77.53 \pm 2.84$ & 80.57 & $81.81 \pm 0.57$ & 82.94 & $83.08 \pm 1.24$ & 85.17 \\
\hline DJ-RFDL & $\mathbf{7 3 . 1 2} \pm \mathbf{0 . 8 7}$ & $\mathbf{7 4 . 5 2}$ & $\mathbf{7 9 . 3 5 \pm 2 . 1 3}$ & $\mathbf{8 1 . 6 1}$ & $\mathbf{8 2 . 9 4 \pm 0 . 8 6}$ & $\mathbf{8 4 . 0 0}$ & $\mathbf{8 4 . 2 0 \pm 1 . 0 5}$ & $\mathbf{8 5 . 5 8}$ \\
\hline \hline
\end{tabular}

In addition, to evaluate our DJ-RFDL with varying size $K$ of dictionary, we randomly select 20 digit images per class as the training set and select the dictionary size as $K=40,80,120,160$, 200. The results are shown in Fig. 8(d). We see that the average rate of each method is increased with the increasing numbers of atoms. DJ-RFDL can outperform its other competitors in most cases, and works steady with varying dictionary sizes.

Recognition results on MNIST database. We also examine each method for recognizing the handwritten digits of MNIST. The MINIST handwritten database has a training set of 60000 samples and a test set of 10000 samples, where each digital image has $28 \times 28$ pixels, which can be represented by using a 784-dimensional vector. In this present work, the training set is employed and we extract random features with the dimension being 100 when evaluating the performance of each method. In our simulations, the number of training samples per digit class is set to 60 (totally 600 training samples) and test on the rest. The classification results are described in Table VIII. We can observe that our proposed formulation can deliver the enhanced results compared with the other related methods. 
TABLE VII: Performance comparison of each algorithm on the OHD database.

\begin{tabular}{|c|c|c|c|c|c|c|c|c|}
\hline \multirow{2}{*}{$\begin{array}{l}\text { Setting } \\
\text { Method }\end{array}$} & \multicolumn{2}{|c|}{ OHD (3 train) } & \multicolumn{2}{|c|}{ OHD (6 train) } & \multicolumn{2}{|c|}{ OHD (9 train) } & \multicolumn{2}{|c|}{ OHD (12 train) } \\
\hline & Mean \pm STD & Best & Mean \pm STD & Best & Mean \pm STD & Best & Mean \pm STD & Best \\
\hline IRPCA & $64.13 \pm 3.97$ & 70.57 & $69.67 \pm 3.53$ & 75.85 & $78.23 \pm 2.54$ & 81.65 & $83.03 \pm 1.73$ & 86.04 \\
\hline LatLRR & $64.06 \pm 4.50$ & 71.32 & $76.96 \pm 2.12$ & 80.75 & $79.61 \pm 1.70$ & 82.14 & $81.53 \pm 2.13$ & 84.00 \\
\hline rLRR & $68.41 \pm 2.74$ & 72.99 & $78.84 \pm 2.15$ & 82.48 & $81.36 \pm 1.93$ & 83.62 & $84.97 \pm 0.76$ & 85.67 \\
\hline KSVD & $61.67 \pm 4.56$ & 67.47 & $67.21 \pm 3.28$ & 70.84 & $72.17 \pm 3.22$ & 75.73 & $76.89 \pm 2.94$ & 80.31 \\
\hline I-LSPFC & $72.46 \pm 4.26$ & 79.75 & $78.59 \pm 3.77$ & 81.52 & $80.29 \pm 3.62$ & 85.13 & $83.12 \pm 1.64$ & 84.73 \\
\hline J-RFDL & $73.40 \pm 0.47$ & 74.22 & $79.24 \pm 0.42$ & 80.01 & $82.54 \pm 0.33$ & 83.15 & $85.22 \pm 0.21$ & 85.82 \\
\hline DLSI & $60.43 \pm 4.41$ & 65.72 & $68.43 \pm 3.26$ & 71.24 & $73.32 \pm 3.58$ & 777.51 & $78.56 \pm 3.24$ & 82.37 \\
\hline ADDL & $75.26 \pm 2.34$ & 79.33 & $79.67 \pm 1.89$ & 81.80 & $82.87 \pm 1.42$ & 84.46 & $85.51 \pm 0.35$ & 86.21 \\
\hline LC-KSVD1 & $62.71 \pm 2.34$ & 65.02 & $68.75 \pm 4.21$ & 73.06 & $72.69 \pm 4.68$ & 78.34 & $77.36 \pm 3.27$ & 82.23 \\
\hline LC-KSVD2 & $63.69 \pm 2.11$ & 66.47 & $69.95 \pm 3.95$ & 74.21 & $75.55 \pm 3.68$ & 80.43 & $78.12 \pm 3.62$ & 82.31 \\
\hline DPL & $72.24 \pm 4.34$ & 77.36 & $79.62 \pm 2.89$ & 82.39 & $81.66 \pm 1.84$ & 83.01 & $84.74 \pm 1.62$ & 86.16 \\
\hline D-KSVD & $62.57 \pm 3.52$ & 67.23 & $68.26 \pm 3.21$ & 71.26 & $73.74 \pm 2.82$ & 76.52 & $78.91 \pm 2.11$ & 80.83 \\
\hline D-LSPFC & $74.28 \pm 3.76$ & 79.00 & $80.64 \pm 1.94$ & 83.92 & $81.18 \pm 2.45$ & 85.18 & $85.43 \pm 0.94$ & 86.60 \\
\hline LRSDL & $68.94 \pm 4.37$ & 72.46 & $75.81 \pm 2.64$ & 79.18 & $79.63 \pm 2.57$ & 82.11 & $81.28 \pm 1.89$ & 84.20 \\
\hline DJ-RFDL & $75.91 \pm 0.25$ & 76.45 & $80.75 \pm 0.18$ & 80.99 & $83.25 \pm 0.16$ & 83.51 & $87.60 \pm 0.14$ & 87.82 \\
\hline
\end{tabular}

TABLE VIII: Performance comparison of each algorithm on the MNIST handwriting digit database.

\begin{tabular}{|c|c|}
\hline Evaluated Methods & Mean \pm STD \\
\hline \hline IRPCA (600 training samples ) & $65.55 \pm 2.17$ \\
\hline LatLRR (600 training samples) & $64.84 \pm 1.42$ \\
\hline rLRR (600 training samples) & $68.62 \pm 1.32$ \\
\hline KSVD (600 training samples) & $66.58 \pm 1.42$ \\
\hline I-LSPFC (600 training samples) & $68.24 \pm 2.11$ \\
\hline J-RFDL(600 training samples) & $\mathbf{7 0 . 9 3} \pm 1.97$ \\
\hline \hline DLSI (600 training samples) & $70.22 \pm 1.88$ \\
\hline ADDL (600 training samples) & $74.33 \pm 2.21$ \\
\hline LC-KSVD1 (600 training samples) & $70.11 \pm 2.58$ \\
\hline LC-KSVD2 (600 training samples) & $72.55 \pm 2.37$ \\
\hline DPL (600 training samples) & $73.81 \pm 1.47$ \\
\hline D-KSVD (600 training samples) & $72.41 \pm 2.32$ \\
\hline D-LSPFC (600 training samples) & $73.24 \pm 1.79$ \\
\hline LRSDL (600 training samples) & $73.41 \pm 2.49$ \\
\hline DJ-RFDL (600 training samples) & $\mathbf{7 6 . 7 6} \pm 1.36$ \\
\hline \hline
\end{tabular}

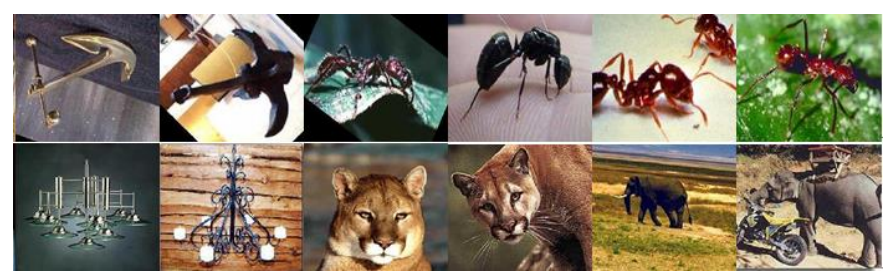

Fig. 10: Image examples of the Caltech101 database.

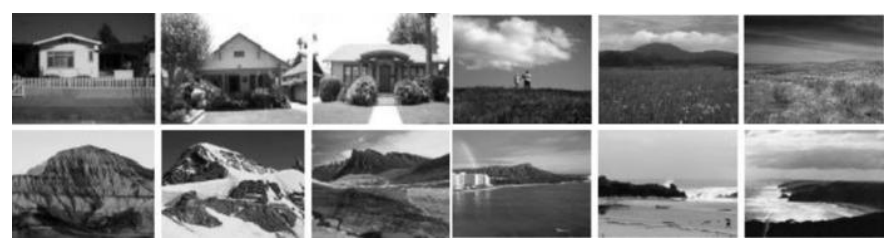

Fig. 11: Image examples of the fifteen natural scene categories database.

\section{G. Natural Object Image Recognition}

In this simulation, we evaluate each algorithm for recognizing the object images of the popular Caltech101 database [62]. This database has 9144 images, consisting of 101 object classes and 1 background class. The number of images in each class varies from 31 to 800 and the size of each image is roughly $300 \times 200$ pixels. Some image examples are show in Fig.10. In this study, we evaluate each method using deep convolutional features,
TABLE IX: Performance comparison of each algorithm on the Caltech101 database.

\begin{tabular}{|c|c|}
\hline Evaluated Methods & Mean \pm STD \\
\hline \hline IRPCA (10 training samples per class) & $70.53 \pm 1.89$ \\
\hline LatLRR (10 training samples per class) & $71.48 \pm 2.11$ \\
\hline rLRR (10 training samples per class) & $74.89 \pm 1.58$ \\
\hline KSVD (10 training samples per class) & $75.11 \pm 1.55$ \\
\hline I-LSPFC (10 training samples per class) & $74.71 \pm 1.72$ \\
\hline J-RFDL(10 training samples per class) & $\mathbf{7 6 . 0 0 \pm 1 . 5 1}$ \\
\hline \hline DLSI (10 training samples per class) & $75.43 \pm 2.81$ \\
\hline ADDL (10 training samples per class) & $84.23 \pm 1.57$ \\
\hline LC-KSVD1 (10 training samples per class) & $76.30 \pm 1.48$ \\
\hline LC-KSVD2(10 training samples per class) & $77.94 \pm 1.67$ \\
\hline DPL (10 training samples per class) & $83.45 \pm 1.49$ \\
\hline D-KSVD (10 training samples per class) & $76.71 \pm 1.53$ \\
\hline D-LSPFC (10 training samples per class) & $82.91 \pm 1.35$ \\
\hline LRSDL (10 training samples per class) & $83.88 \pm 1.59$ \\
\hline DJ-RFDL (10 training samples per class) & $\mathbf{8 5 . 0 5} \pm \mathbf{1 . 4 2}$ \\
\hline \hline
\end{tabular}

TABLE X: Performance comparison of each methods on the fifteen natural scene categories database.

\begin{tabular}{|c|c|}
\hline Evaluated Methods & Mean \pm STD \\
\hline \hline IRPCA (20 training samples per class) & $49.87 \pm 1.76$ \\
\hline LatLRR (20 training samples per class) & $50.29 \pm 1.88$ \\
\hline rLRR (20 training samples per class) & $51.23 \pm 1.77$ \\
\hline KSVD (20 training samples per class) & $52.63 \pm 1.28$ \\
\hline I-LSPFC (20 training samples per class) & $52.23 \pm 1.45$ \\
\hline J-RFDL(20 training samples per class) & $\mathbf{5 4 . 7 9} \pm \mathbf{1 . 3 1}$ \\
\hline \hline DLSI (20 training samples per class) & $56.11 \pm 1.33$ \\
\hline ADDL (20 training samples per class) & $63.18 \pm 1.10$ \\
\hline LC-KSVD1 (20 training samples per class) & $57.21 \pm 1.37$ \\
\hline LC-KSVD2(20 training samples per class) & $59.39 \pm 2.11$ \\
\hline DPL (20 training samples per class) & $62.78 \pm 1.27$ \\
\hline D-KSVD (20 training samples per class) & $58.19 \pm 1.66$ \\
\hline D-LSPFC (20 training samples per class) & $61.27 \pm 2.14$ \\
\hline LRSDL (20 training samples per class) & $62.38 \pm 1.77$ \\
\hline DJ-RFDL (20 training samples per class) & $\mathbf{6 3 . 6 8} \pm \mathbf{1 . 5 8}$ \\
\hline \hline
\end{tabular}

and we employ the classical Alexnet framework [64] to extract the deep features from original images. To extract deep features, the input size of images is set according to the requirement Alexnet [64] and the features of the penultimate full connection layer ('fc7') are evaluated. Finally, the dimension of extracted features is 4096. Then, we can construct a feature matrix based 
on extracted deep features for representation learning by each method for fair comparison. The number of training samples is set to 10 per class and we test on the rest. The dictionary size is set to the number of training samples. The averaged recognition results are described in Table IX. We see from the results that our methods deliver higher recognition results.

\section{H. Natural Scene Category Recognition}

We also evaluate each method for recognizing the natural scene categories. The fifteen natural scene categories database [63] is involved in this study, which contains 15 natural scenes, that is, suburb, open country, mountain, coast, highway, forest, store, office, kitchen, industrial, living room, bedroom, tall building, street and inside city. Each category has 200 to 400 images that are about $250 \times 300$ resolution. Some image examples are show in Fig.11. We also use the deep features exacted by Alexnet [64] for recognition, and select 20 samples per category as training set. Since the inputs of Alexnet are RGB images, in this study we convert each gray image into a RGB image by copying the gray image into each of the three channels of RGB space. The recognition results are shown in Table $X$, where we find that our models deliver higher accuracies in the two groups.

\section{Noisy Image Recognition Evaluation}

We evaluate the robustness of each method using noisy images. The results under different pixel corruptions are computed. The default dictionary size is set to the number of training samples. Three kinds of real images are evaluated.

(1) Face recognition with pixel corruptions. UMIST face database [49] is evaluated. We fix the number of training face images of each class to 6 and vary the percentages of corrupted pixels. Fig.12 shows the result of each method as a function of random corruptions, where the results are averaged based on 10

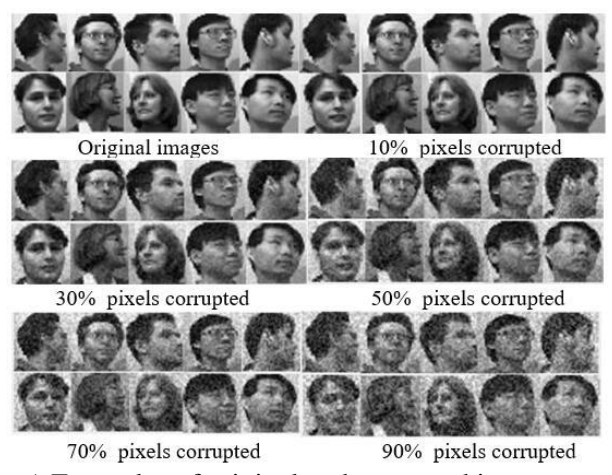

a) Examples of original and corrupted images

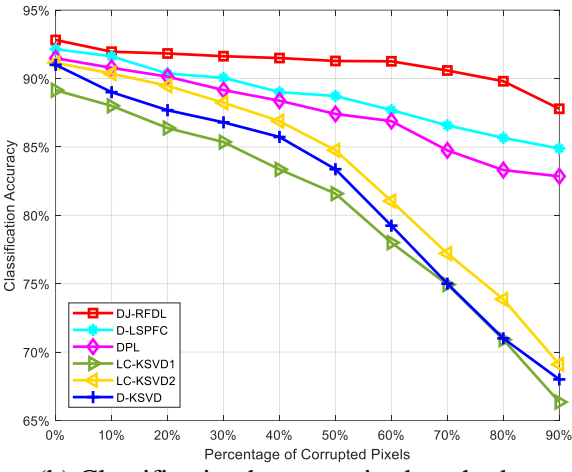

(b) Classification by supervised methods

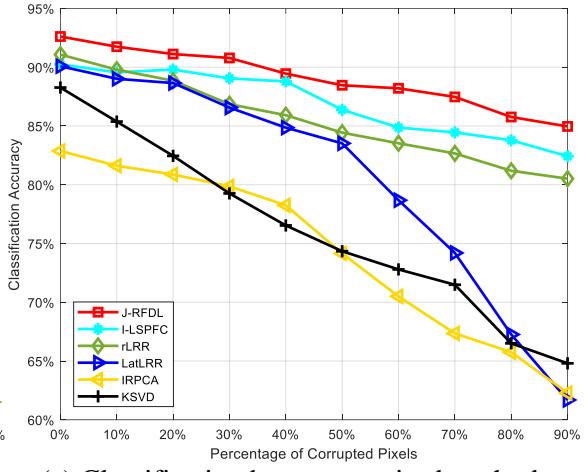

(c) Classification by unsupervised methods

Fig. 12: Handwritten digit recognition results of each algorithm under different levels of pixel corruptions on the UMIST face database.

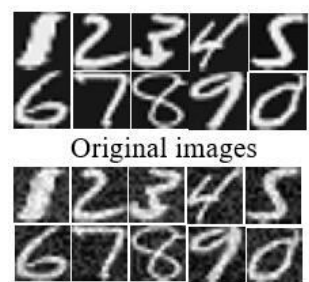

$30 \%$ pixels corrupted

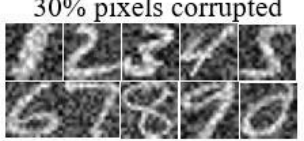

$70 \%$ pixels corrupted
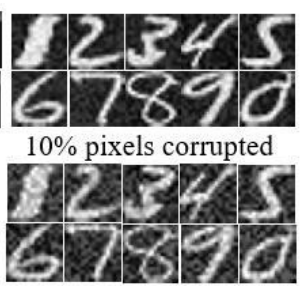

$50 \%$ pixels corrupted

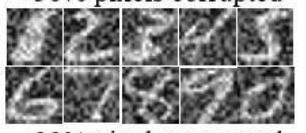

and corrupted images

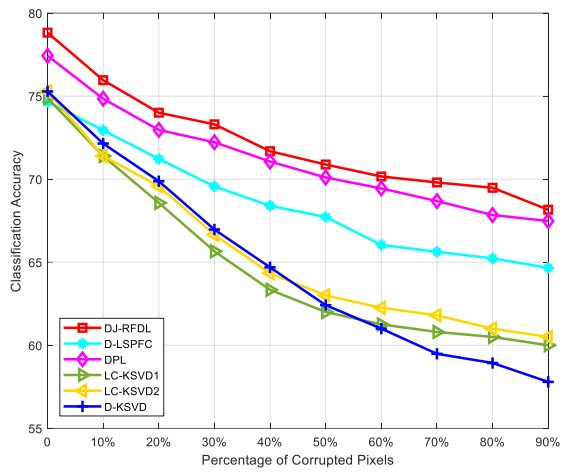

(b) Classification by supervised methods

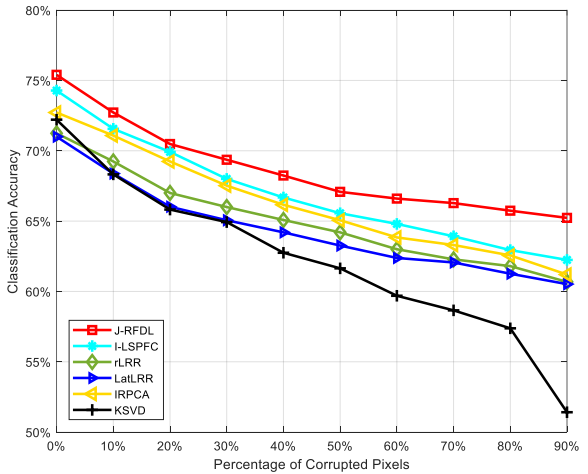

(c) Classification by unsupervised methods

Fig. 13: Handwritten digit recognition results of each algorithm under different levels of pixel corruptions on the USPS digits database.

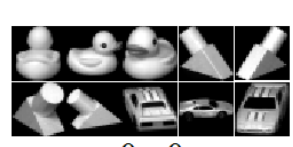

$0 \times 0$

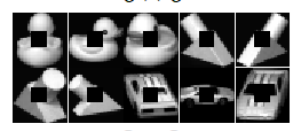

$8 \times 8$

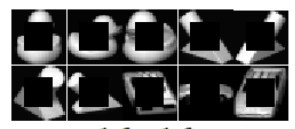

$16 \times 16$

(a) Examples of occupied images

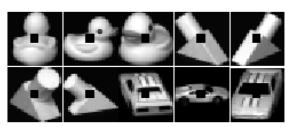

$4 \times 4$

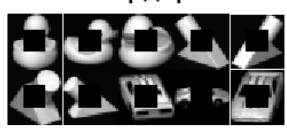

$12 \times 12$

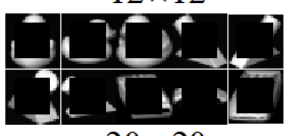

$20 \times 20$

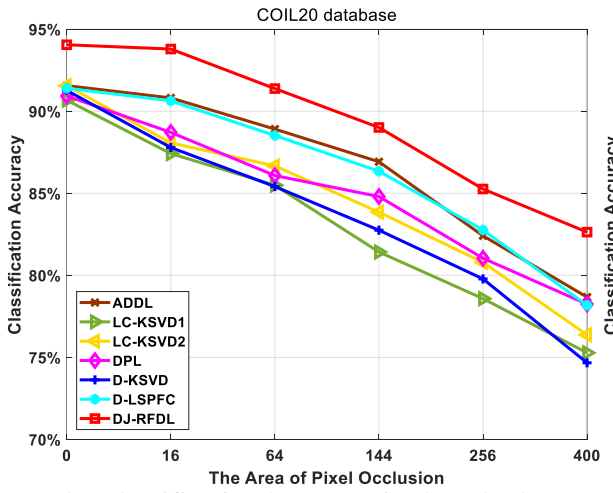

(b) Classification by supervised methods

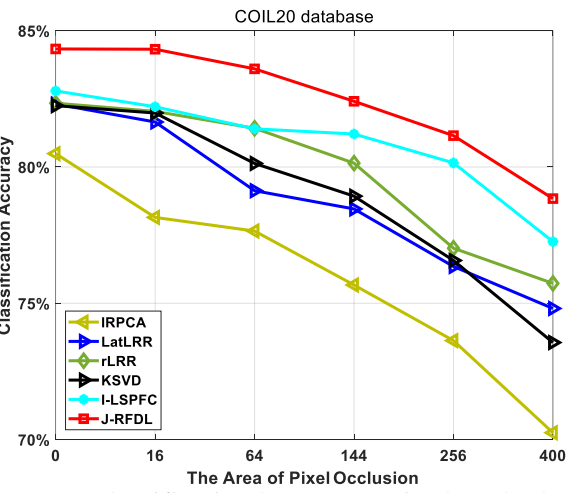

(c) Classification by unsupervised methods

Fig. 14: Object recognition results of each algorithm under different areas of block occlusion on the COIL20 object database. 
random splits of training/testing images and 10 random pixel selections. We can see that the performance of each method is decreased with the increasing percentages of corruptions, and the results of our methods usually go down slower than other methods as the percentage of corruptions is increased. For the supervised group, DPL and D-LSPFC are more robust to the noise than other methods. The results of D-KSVD, LC-KSVD1 and LC-KSVD2 are decreased rapidly when the percentage of the corrupted pixels is larger than 50\%. For unsupervised group, J-RFDL achieves higher and more stable results than the other compared methods. rLRR and I-LSPFC also perform well.

(2) Handwritten recognition with pixel corruptions. We also examine J-RFDL and DJ-RFDL for classifying the handwritten digits under noisy case on USPS database [23]. We select 20 images per digit as training set and test on the rest, and vary the percentage of corrupted pixels from $10 \%$ to $90 \%$. Fig. 13 shows the averaged results over 10 random splits, from which we find that our J-RFDL and DJ-RFDL outperform other competitors in each group under different percentages of corrupted pixels. For the supervised group, DPL also works well, followed by D-LSPFC, and both are superior to other remaining methods. For the unsupervised group, I-LSPFC and IRPCA also deliver better results, and rLRR also obtains better results than LatLRR. $\mathrm{K}-\mathrm{SVD}$ is still the worst method in most cases.

(3) Object recognition with missing pixel values. We test each approach for classifying the object images under noisy case on the COIL-20 database. We choose 20 images per object as training set and test on the rest. In this study, each method performs classification over the images with different areas of missing pixel values by block occlusion similarly as [51]. More specifically, we define areas with different sizes and then set the pixel values of these areas to 0, i.e., missing values. Fig.14 shows the results of each method as a function of the areas of block occlusion, where some examples of the occupied images are also shown. From the results, we see that the performance of each algorithm is still decreased with the increasing numbers of missing values. Our J-RFDL and DJ-RFDL can deliver higher results than other compared methods in each group.

\section{J. Further Evaluation on Classification}

We also conduct a simulation to compare our algorithms with several deep networks-based methods, including Deep Belief Network (DBN) [53], Discriminative deep DBN (DDBN) [54], Deep Dictionary Learning (DDL) [55] and Supervised Deep Dictionary Learning (SDDL) [56]. Following the procedures in [56], YaleB face database [61] and AR face database [60] are employed as examples. More specifically, we select one half of the images for training and the remaining ones for testing on YaleB. For AR, we randomly chose 20 samples per class for training and test on the rest. The averaged classification results

TABLE XI: Comparison Results on YaleB and AR.

\begin{tabular}{|c|c|c|}
\hline Methods & YaleB face & AR face \\
\hline DBN & $34.91 \pm 4.14$ & $43.62 \pm 4.32$ \\
\hline DDBN & $38.20 \pm 4.57$ & $60.34 \pm 5.68$ \\
\hline DDL & $92.66 \pm 5.11$ & $93.35 \pm 5.26$ \\
\hline SDDL (1-layer) & $89.45 \pm 5.52$ & $91.13 \pm 5.61$ \\
\hline SDDL (2-layer) & $90.31 \pm 5.27$ & $91.17 \pm 5.28$ \\
\hline SDDL (3-layer) & $96.50 \pm 5.36$ & $94.57 \pm 5.41$ \\
\hline SDDL (4-layer) & $96.16 \pm 5.57$ & $94.24 \pm 5.62$ \\
\hline DJ-RFDL & $\mathbf{9 7 . 5 0} \pm \mathbf{3 . 3 1}$ & $\mathbf{9 8 . 3 3 + 3 . 2 7}$ \\
\hline
\end{tabular}

are shown in Table XI, where the results of DBN, DDBN, DDL and SDDL are directly adopted from [56]. From the results, we can find that our proposed DJ-RFDL still outperforms the deep networks-based methods for face recognition. Recent SDDL method obtains comparable results to our method.

\section{CONCLUSION AND FUTURE WORK}

We have discussed the problem of recovering the hybrid salient low-rank and sparse representation through robust dictionary learning in a factorized compressed feature space, and present a robust factorization and projective dictionary learning model. J-RFDL clearly unifies the robust matrix factorization, robust dictionary learning and the hybrid salient representation. More specifically, our method aims at improving the representation ability by enhancing the robustness of the reconstruction metric to outliers and noise, encoding the reconstruction errors more accurately and obtaining the hybrid salient coefficients that can best represent given data. By combining the classification error over hybrid salient coefficients into J-RFDL to form a unified model, we also present a discriminative J-RFDL model.

We have examined the effectiveness of our methods based on several public databases. The recognition results based on the original and corrupted samples show the superior performance of our methods, compared with several related approaches. In future, we will extend our methods to semi-supervised scenario by using labeled and unlabeled data. Two possible approaches can be considered. (1) We can compute the salient features and hybrid salient coefficients by minimizing the reconstruction error based on labeled samples within each class, and as the same time minimize the reconstruction error over all classes as a whole to preserve the global structures; (2) We can re-define the initial label matrix $H=\left[h_{1}, h_{2}, \ldots h_{N}\right] \in \mathbb{R}^{c \times N}$ based on labeled and unlabeled training samples (i.e., for each training sample $x_{j}$, $h_{i, j}=1$ if $x_{j}$ belongs to the class $i, 1 \leq i \leq c$, and else $\left.h_{i, j}=0\right)$, and at the same time consider propagating the label information from labeled data to the unlabeled data. In addition, the optimal determination of dictionary size still remains an open problem and will also be investigated in future work.

\section{ACKNOWLEDGMENT}

The authors would like to express sincere thanks to reviewers for their insightful comments, making our manuscript a higher standard. This work is partially supported by National Natural Science Foundation of China (61672365, 61732008, 61725203, 61622305, 61871444 and 61572339), and the Fundamental Research Funds for the Central Universities of China (JZ2019HGPA0102). Dr. Zhao Zhang is the corresponding author.

\section{REFERENCES}

[1] Y. Bengio, A. C. Courville, and P. Vincent, "Representation Learning: A Review and New Perspectives. IEEE Trans. Pattern Anal. Mach. Intell., vol.35, no.8, pp.1798-1828, 2013.

[2] A. Behl, D. Paschalidou, S. Donné, A. Geiger, "PointFlowNet: Learning Representations for Rigid Motion Estimation From Point Clouds," In: Proceedings of the IEEE Conference on Computer Vision and Pattern Recognition pp.7962-7971, 2019.

[3] Z. Zhang, J. Ren, S. Li, R. Hong, Z. Zha and M. Wang, "Robust Subspace Discovery by Block-diagonal Adaptive Locality-constrained Representation," In: Proceedings of the ACM International Conference on Multimedia, Nice, France, pp.1569-1577, Oct 2019.

[4] J. Wright, Y. Ma, J. Mairal, "Sparse Representation for Computer Vision and Pattern Recognition," Proceedings of the IEEE, vol.98, no.6, pp.1031-1044, 2010. 
[5] Y. Sun, Z. Zhang, W. Jiang, Z. Zhang, L. Zhang, S. Yan and M. Wang, "Discriminative Local Sparse Representation by Robust Adaptive Dictionary Pair Learning," IEEE Transactions on Neural Networks and Learning Systems, Nov 2019.

[6] Z. Zhang, J. Ren, W. Jiang, Z. Zhang, R. Hong, S. Yan and M. Wang, "Joint Subspace Recovery and Enhanced Locality Driven Robust Flexible Discriminative Dictionary Learning," IEEE Transactions on Circuits and Systems for Video Technology, June 2019.

[7] J. Mairal, F. Bach and J. Ponce, "Task-driven dictionary learning," IEEE transactions on pattern analysis and machine intelligence, vol.34, no.4 pp.791-804, 2012.

[8] M. Elad and M. Aharon, "Image denoising via sparse and redundant representations over learned dictionaries," IEEE Transactions on Image Processing, vol.15, no.12, pp.3736-3745, 2006.

[9] G. Liu, Z. Lin, and S. C. Yan, "Robust Recovery of Subspace Structures by Low-Rank Representation," IEEE Transactions on Pattern Analysis and Machine Intelligence, vol.35, no.1, pp.171-184, 2013.

[10] Z. Zhang, C L. Liu, and M. B. Zhao, "A sparse projection and low-rank recovery framework for handwriting representation and salient stroke feature extraction," ACM TIST, vol.6, no.1, 2015.

[11] D. D. Lee and H. S. Seung, "Learning the parts of objects by nonnegative matrix factorization," Nature, vol. 401, no.6755, pp. 788-791, 1999.

[12] Z. Zhang, Y. Zhang, S.Li, G. Liu, D. Zeng, S. Yan, M. Wang, "Flexible Auto-weighted Local-coordinate Concept Factorization: A Robust Framework for Unsupervised Clustering," IEEE Trans. on Knowledge and Data Engineering, Sep 2019.

[13] E. J. Candès, X. Li and Y. Ma, "Robust principal component analysis?," Journal of the ACM, vol.58, no.3, pp.1-11, 2011.

[14] B. K. Bao, G. C. Liu, and C. Xu, "Inductive robust principal component analysis," IEEE Trans.on Image Proc., vol.21, no.8, pp.3794-3800, 2012.

[15] G. C. Liu, and S. C. Yan, "Latent low-rank representation for subspace segmentation and feature extraction," Proc. IEEE Int. Conf. on. Computer Vision, Barcelona, Spain, pp.1615-1622, 2011.

[16] Z. Zhang, S. C. Yan, and M. B. Zhao, "Similarity preserving low-rank representation for enhanced data representation and effective subspace learning," Neural Networks, vol.53, pp. 81-94, 2014.

[17] S. Gu, L. Zhang, and W. Zuo, "Projective dictionary pair learning for pattern classification," NIPS, pp.793-801, 2014.

[18] Z. Zhang, F. Li, M. Zhao, L. Zhang, S. Yan, “Joint low-rank and sparse principal feature coding for enhanced robust representation and visual classification," IEEE Transactions on Image Processing, vol.25, no.6, pp.2429-2443, 2016.

[19] Z. Lin, M. Chen, L. Wu, and Y. Ma, "The augmented Lagrange multiplier method for exact recovery of corrupted low-rank matrices," Tech. Tep. UILU-ENG-09-2215, 2009.

[20] M. Aharon, M. Elad, A. Bruckstein, Y. Katz, "K-SVD: An Algorithm for Designing Overcomplete Dictionaries for Sparse Representation," IEEE Transactions on Signal Processing, vol.54, no.1, pp.4311-4322, 2006.

[21] I. Ramirez, P. Sprechmann and G. Sapiro, "Classification and Clustering via dictionary learning with structured incoherence and shared features," Proc. CVPR, CA, USA, pp. 3501-3508, 2010.

[22] Z. Zhang, W. Jiang, J. Qin, L. Zhang, F. Li, M. Zhang, S. C. Yan, "Jointly Learning Structured Analysis Discriminative Dictionary and Analysis Multiclass Classifier," IEEE Trans. on Neural Networks and Learning Systems, vol.29, iss.8, pp.3798-3814, 2018.

[23] J. Hull, "A database for handwritten text recognition research," IEEE Transactions on pattern analysis and machine intelligence, vol.16, no.5, pp.550-554, 1994.

[24] C. P. Hou, F. P. Nie, X. L. Li, D.Y. Yi, and Yi Wu, "Joint Embedding Learning and Sparse Regression: A Framework for Unsupervised Feature Selection," IEEE Trans. on Cybernetics, vol.44, no.6, pp.793- 804, 2014.

[25] Q. Zhang and B. Li, "Discriminative K-SVD for Dictionary Learning in Face Recognition," Proc. IEEE Conf. Computer Vision and Pattern Recognition, San Francisco, CA, USA, pp. 2691-2698, 2010.

[26] Z. L. Jiang, Z. Lin, L. S. Davis, "Learning a Discriminative Dictionary for Sparse Coding via Label Consistent K-SVD,"Proc. IEEE Conference on Computer Vision and Pattern Recognition, Providence, 2011.

[27] Z. L. Jiang, Z. Lin, and L. S. Davis, "Label Consistent K-SVD: Learning A Discriminative Dictionary for Recognition," IEEE Trans. on Pattern Analysis and Machine Intelligence, vol.35. no.11, pp.2651-2664, 2013.

[28] L. Ma, C.H. Wang, B.H. Xiao, W. Zhou, "Sparse Representation for Face Recognition based on Discriminative Low-Rank Dictionary Learning," In Proceedings of the IEEE Conference on Computer Vision and Pattern Recognition, Providence, RI, pp. 2586-2593, 2012.
[29] M. Yang, L. Zhang, X. Feng, and D. Zhang, "Fisher Discrimination Dictionary Learning for Sparse Representation," In: Proceedings of the International Conf. on Computer Vision, Barcelona, Spain, 2011.

[30] M. Yang, L. Zhang, X. Feng, D. Zhang, "Sparse Representation Based Fisher Discrimination Dictionary Learning for Image Classification," International Journal of Computer Vision, vol.109, pp.209-232 2014.

[31] L.Y. Li, S. Li Y. Fu, "Learning low-rank and discriminative dictionary for image classification," Image and Vision Computing, vol.32, no.10, pp.814-823, 2014

[32] Y. Zhang, Z. L. Jiang, and L. Davis, "Learning structured low-rank representations for image classification," In Proceedings of IEEE Conf. on Computer Vision and Pattern Recognition, Portland, 2013.

[33] J. Chen and Z. Yi, "Sparse representation for face recognition by discriminative low-rank matrix recovery," J. Vis. Commun. Image R., vol.25, no.5, pp.763-773, 2014.

[34] J. Xie, J. Yang, J. Qian, Y. Tai, H. Zhang, "Robust Nuclear Norm-Based Matrix Regression With Applications to Robust Face Recognition," IEEE Trans. Image Processing, vol.26, no.5, pp.2286-2295, 2017.

[35] Y. Yang, H. T. Shen, Z. G. Ma, Z. Huang, and X. F. Zhou, "L2, 1-Norm Regularized Discriminative Feature Selection for Unsupervised Learning," In: Proceeding of the International Joint Conferences on Artificial Intelligence, vol.22, no.1, pp.1589, 2011.

[36] Z. Zhang, L. Zhang, M. B. Zhao, W. M. Jiang, Y. C. Liang, and F. Z. Li, "Semi-Supervised Image Classification by Nonnegative Sparse Neighborhood Propagation," In: Proceedings of the ACM International Conference on Multimedia Retrieval, Shanghai, China, pp.139-146, 2015.

[37] F. P. Nie, H. Huang, X. Cai, and C. Ding, "Efficient and Robust Feature Selection via Joint L21-Norms Minimization," In: Advances in Neural Information Processing Systems, Vancouver, pp.1813-1821, 2010.

[38] Z. Zhang, Y. Sun, Z. Zhang, Y. Wang, G. Liu and M. Wang, "Learning Structured Twin-Incoherent Twin-Projective Latent Dictionary Pairs for Classification," In: Proceedings of the IEEE International Conference on Data Mining (ICDM), Beijing, China, Oct 2019.

[39] J. Xie, J. Yang, J. Qian, Y. Tai, H. Zhang, "Robust Nuclear Norm-Based Matrix Regression With Applications to Robust Face Recognition," IEEE Trans. Image Processing, vol.26, no.5, pp.2286-2295, 2017.

[40] I. Ramirez, P. Sprechmann, and G. Sapiro, "Classification and clustering via dictionary learning with structured incoherence and shared features," in IEEE Conf. Computation. Vis. Pattern Recognition, San Francisco, CA, USA, pp. 3501-3508, 2010.

[41] D. D. Lee and H. S. Seung, "Learning the parts of objects by nonnegative matrix factorization," Nature, vol. 401, no.6755, pp. 788-791, 1999.

[42] Z. Zhang, Y. Zhang, G. Liu, J. Tang, S. Yan and M. Wang, "Joint Label Prediction based Semi-Supervised Adaptive Concept Factorization for Robust Data Representation," IEEE Trans. on Knowledge and Data Engineering, Jan 2019.

[43] S. A. Nene, S. K. Nayar and H. Murase, "Columbia Object Image Library (COIL-20),", Technical Report CUCS-005-96, February 1996.

[44] T. Sim, S. Baker, M. Bsat, "The CMU pose, illuminlation, and expression database," IEEE Trans. on Pattern Analysis and Machine Intelligence, vol.25, no.12, pp.1615-1618, 2003.

[45] C. Kaynak "Methods of Combining Multiple Classifiers and Their Appl ications to Handwritten Digit Recognition,"MSc Thesis, Institute of Gra duate Studies in Science and Engineering, Bogazici University, 1995.

[46] T. H. Cormen, "Introduction to algorithms,"MIT press, 2009.

[47] M. A. Schmitz, M. Heitz, N. Bonneel, F. Ngole, D. Coeurjolly, M. Cuturi, Gabriel Peyré, J.L. Starck, "Wasserstein Dictionary Learning: Optimal Transport-Based Unsupervised Nonlinear Dictionary Learning," SIAM Journal on Imaging Sciences, vol.11, no.1, pp.643-678, 2018.

[48] D.S. Pham and V. Svetha, "Joint learning and dictionary construction for pattern recognition," in Proc. IEEE Conf. Comput. Vis. Pattern Recognit., Anchorage, AK, USA, Jun, pp. 23-28, 2008.

[49] D. Graham and N. Allinson, "Characterizing virtual eigensignatures for general purpose face recognition," in Face Recognition: From Theory to Application (NATO ASI Series F, Computer and Systems Sciences), Springer, Berlin, Heidelberg, vol. 163, pp. 446-456, 1998.

[50] T. Oh, Y. Matsushita, Y. Tai, I. S. Kweon, "Fast Randomized Singular Value Thresholding for Low-Rank Optimization," IEEE Trans. Pattern Anal. Mach. Intell., vol.40, no.2), pp.376-391, 2018.

[51] X. Liu, X. Zhu, M. Li, L. Wang, E. Zhu, T. Liu, and W. Gao, "Multiple kernel k-means with incomplete kernels," IEEE Transactions on Pattern Analysis and Machine Intelligence (Early Access ), 2019.

[52] T. H. Vu and V. Monga, "Fast low-rank shared dictionary learning for image classification," IEEE Transactions on Image Processing, vo.26, no.11, pp. 5160-5175, 2017. 
[53] G.E. Hinton, "Deep belief networks," Scholarpedia, vol.4, no.5, pp.5947, 2009.

[54] Y. Liu, S. Zhou, Q.Chen, "Discriminative deep belief networks for visual data classification," Pattern Recognition, vol.44, pp.2287-2296, 2011

[55] S. Tariyal, A. Majumdar, R.Singh, M.Vatsa, "Deep dictionary networks," IEEE Access, vol.4, pp.10096-109, 2016.

[56] V. Singhal, A. Majumdar "Supervised Deep Dictionary Learning for Single Label and Multi-Label Classification," In: Proceedings of IEEE International Joint Conference on Neural Networks, pp.1-7, 2018.

[57] B. Weyrauch, J. Huang, B. Heisele, V.Blanz, "Component-based Face Recognition with 3D Morphable Models," In: Proc. 1st IEEE workshop on Face Processing in Video, Washington, D.C, 2004.

[58] Y. L. Cun, L. Bottou, Y. Bengio, P. Haffner. "Gradient-based learning applied to document recognition," Proceedings of the IEEE, vol.86, no.11, pp.2278-2324, 1998.

[59] J. Ren, Z. Zhang, S. Li, G. Liu, M. Wang, and S. Yan, "Robust Projective Low-Rank and Sparse Representation by Robust Dictionary Learning," In Proceedings of the 24th International Conference on Pattern Recognition, Beijing, China, April 2018

[60] A. Martinez and R. Benavente, "The AR Face Database," CVC Technical Report 24, 1998.

[61] A. Georghiades, P. Belhumeur, and D. Kriegman, "From Few to Many: Illumination Cone Models for Face Recognition under Variable Lighting and Pose," IEEE Trans. Pattern Analysis and Machine Intelligence, vol. 23, no. 6, pp. 643-660, 2001.

[62] P. Perona, R. Fergus and F. Li, "Learning generative visual models from few training samples: An incremental Bayesian approach tested on 101 object categories," Computer Vision and Image Understanding, vol.106, no. 1 , pp.59-70, 2007

[63] S. Lazebnik, C. Schmid and J. Ponce, "Beyond bags of features: Spatial pyramid matching for recognizing natural scene categories," in proc. IEEE CVPR, New York, NY, USA, pp.2169-2178, 2006.

[64] A. Krizhevsky, I. Sutskever, and G. E. Hinton, "Imagenet classification with deep convolutional neural networks," In: Advances in Neural Information Processing Systems, pp.1097-1105, 2012.

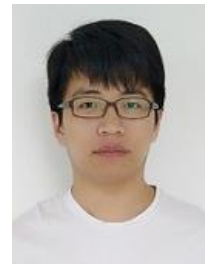

Jihuan Ren is currently working toward the research degree at School of Computer Science and Technology, Soochow University, Suzhou 215006, China. His current research interests include pattern recognition, machine learning, data mining and their applications. Specifically, he is very interested in designing the advanced low-rank coding and dictionary learning algorithms for the robust image representation and classification.

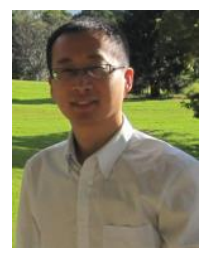

Zhao Zhang (SM'17- ) is a Full Professor in the School of Computer Science, Hefei University of Technology, Hefei, China. He received the Ph.D. degree from the Department of Electronic Engineering at City University of Hong Kong, in 2013. His current research interests include Data Mining \& Machine Learning, Image Processing \& Pattern Recognition. He has authored/co-authored more than 90 technical papers published at prestigious journals and conferences, such as IEEE TIP (6), IEEE TKDE (6), IEEE TNNLS (8), IEEE TSP, IEEE TCSVT, IEEE TCYB, IEEE TBD, IEEE TII (2), ACM TIST, Pattern Recognition (6), Neural Networks (8), Computer Vision and Image Understanding, ACM Multimedia, IJCAI, ICDM (4), SDM, ICASSP and ICMR, etc. Specifically, he has published 26 regular papers in IEEE/ACM Transactions. Dr. Zhang is serving/served as an Associate Editor (AE) for IEEE Access, Neurocomputing and IET Image Processing. Besides, he has been acting as a Senior PC member/Area Chair of IJCAI、ECAI、BMVC and PAKDD, and a PC member for $10+$ popular prestigious conferences (e.g., CVPR、ICCV 、IJCAI、 AAAI ACM MM、ECCV 、ICDM、CIKM and SDM). He is now a Senior Member of the IEEE, and a Senior Member of the CCF.

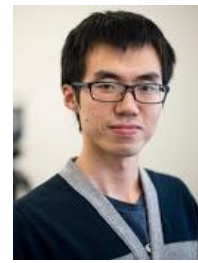

Sheng Li (M'17-SM'19-) received the Ph.D. degree from Northeastern University, Boston, MA, in 2017. He is now a Tenure-Track Assistant Professor in the Department of Computer Science at University of Georgia. He has published over 50 papers at leading conferences and journals. $\mathrm{He}$ has received the best paper awards (or nominations) at SDM 2014, IEEE ICME 2014, and IEEE FG 2013. He is serving on the Editorial Boards of Neural Computing and
Applications, and also serves as an Associate Editor of IEEE Computational Intelligence Magazine, Neurocomputing and IET Image Processing, etc. He has also served as a reviewer for several IEEE Transactions, and program committee member for NIPS, IJCAI, AAAI, and KDD. His research interests include robust machine learning, dictionary learning, visual intelligence, and behavior modeling. He is now a Senior Member of the IEEE.

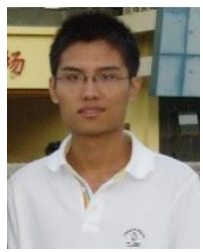

Yang Wang is now a Professor at the Hefei University of Technology, China. He earned the $\mathrm{PhD}$ degree from the University of New South Wales, Australia. He has published over 50 research papers in pattern recognition and machine learning field, such as IEEE TIP, IEEE TNNLS, IEEE TCSVT, IEEE TMM, IEEE TKDE, IEEE TCYB, IJCAI, ACM SIGIR, ACM Multimedia, IEEE ICDM, ACM CIKM, VLDB Journal, Pattern Recognition and Neural Networks. Yang Wang is an Associate Editor for ACM Transactions on Information Systems (ACM TOIS), while served as a Guest Editor on IEEE Multimedia magazine, Pattern Recognition Letters.

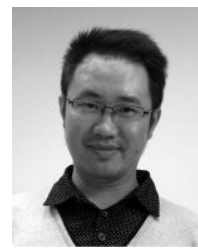

Guangcan Liu (M'11-SM'18- ) received the bachelor's degree in mathematics and the Ph.D. degree in computer science and engineering from the Shanghai Jiao Tong University, Shanghai, China, in 2004 and 2010, respectively. He was a Post-Doctoral Researcher with the National University of Singapore, Singapore, from 2011 to 2012, the University of Illinois at Urbana-Champaign, Champaign, IL, USA, from 2012 to 2013, Cornell University, Ithaca, NY, USA, from 2013 to 2014, and Rutgers University, Piscataway, NJ, USA, in 2014. Since 2014, he has been a Professor with the School of Information and Control, Nanjing University of Information Science and Technology, Nanjing, China. His research interests touch on the areas of pattern recognition and signal processing. He obtained the National Excellent Youth Fund in 2016 and was designated as the global Highly Cited Researchers in 2017. He is now a Senior Member of the IEEE.

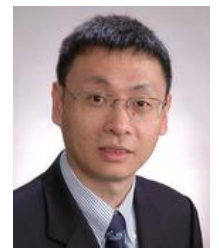

Shuicheng Yan (F'16-) received the Ph.D. degree from the Peking University in 2004. He is now the CTO of Yitu Tech, and also a (Dean's Chair) Associate Professor at the National University of Singapore. His research areas include computer vision, multimedia and machine learning, and he authored/co-authored more than 370 technical papers over a wide range of research topics, with the Google Scholar citations >58, 000 times and H-index-100. He is ISI Highly-cited Researcher of 2014, 2015 and 2016. He is/has been an Associate Editor of IEEE Trans. Knowledge and Data Engineering (TKDE), IEEE Trans. Circuits and Systems for Video Technology (TCSVT), ACM Trans. Intelligent Systems and Technology (TIST), and Journal of Computer Vision and Image Understanding. He is also a Fellow of the IEEE and the IAPR.

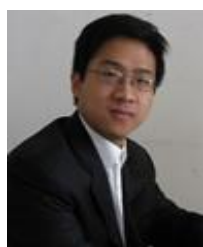

Meng Wang is a full professor in the Hefei University of Technology, China. He received the B.E. degree and Ph.D. degree in the Special Class for the Gifted Young and signal and information processing from the University of Science and Technology of China, Hefei, China, respectively. He previously worked as an associate researcher at Microsoft Research Asia, and then a core member in a startup in Bay area. After that, he worked as a senior research fellow in National University of Singapore. His current research interests include multimedia content analysis, search, mining, recommendation, and large-scale computing. He has authored 6 book chapters and over 100 journal and conference papers in these areas, including TMM, TNNLS, TCSVT, TIP, TOMCCAP, ACM MM, WWW, SIGIR, ICDM, etc. He received the paper awards from ACM MM 2009 (Best Paper Award), ACM MM 2010 (Best Paper Award), MMM 2010 (Best Paper Award), ICIMCS 2012 (Best Paper Award), ACM MM 2012 (Best Demo Award), ICDM 2014 (Best Student Paper Award), PCM 2015 (Best Paper Award), SIGIR 2015 (Best Paper Honorable Mention), IEEE TMM 2015 (Best Paper Honorable Mention), and IEEE TMM 2016 (Best Paper Honorable Mention). He is the recipient of ACM SIGMM Rising Star Award 2014. He is/has been an Associate Editor of IEEE Trans. on Knowledge and Data Engineering (TKDE), IEEE Trans. on Neural Networks and Learning Systems (TNNLS) and IEEE Trans. on Circuits and Systems for Video Technology (TCSVT). He is a senior member of IEEE. 